M. Maruyama

Nagoya Math. J.

Vol. 78 (1980), 65-94

\title{
BOUNDEDNESS OF SEMI-STABLE SHEAVES OF SMALL RANKS
}

\author{
MASAKI MARUYAMA
}

\section{Introduction}

As for the construction of moduli spaces of stable sheaves, the boundedness of semi-stable sheaves is one of the most important questions which are left unanswered. In the case of dimension one, the boundedness was proved by M. F. Atiyah [1]. When the dimension of the base variety is two and the rank is two, F. Takemoto and D. Mumford showed the boundedness independently ([13]). The author proved in [7] that the boundedness holds for every rank in the case of dimension two, and then D. Gieseker gave another proof of it in [3].

In this article, we shall prove the boundedness in some very special cases which are not contained in the above results. The author hopes that his results are enough to give evidences of the boundedness in general cases.

To state our results, let $f: X \rightarrow S$ be a smooth, projective, geometrically integral morphism of noetherian schemes over a ring $\Lambda$ and let $\mathcal{O}_{X}(1)$ be an $f$-very ample invertible sheaf on $X$. Our first main result is

TheOREm 3.1. If $S=\operatorname{Spec}(k)$ with an algebraically closed field $k$, $\operatorname{dim} X \geq 2, E$ is $\mu$-semi-stable (Definition 1.1) and if $r(E)<\operatorname{dim} X$, then for almost all $Y$ in $\left|\mathcal{O}_{X}(1)\right|,\left.E\right|_{Y}$ is $\mu$-semi-stable.

As for the boundedness, we shall consider three statements; $B_{n, r}(\Lambda)$. $B_{n, r}^{\prime}(\Lambda)$ and $B_{n, r}^{\prime \prime}(\Lambda)$ ((3.4.1), (3.4.2) and (3.4.3)). Lemma 3.5 shows that $B_{n, r}(\Lambda)$ implies $B_{n, r}^{\prime \prime}(\Lambda)$ and $B_{n, r}^{\prime}(\Lambda)$ does $B_{n, r}^{\prime \prime}(\Lambda)$. When $B_{n, r}^{\prime \prime}(\Lambda)$ holds for a couple $(n, r)$, we say that the boundedness of semi-stable sheaves holds in the case of dimension $n$ and rank $r$ (in the category of $\Lambda$-schemes). As a direct corollary to Theorem 3.1 , we see that $B_{n, 2}(\Lambda)$ and $B_{n, 2}^{\prime}(\Lambda)$ hold for all $n$ and $\Lambda$ (Theorem 3.11).

In $\S 4$, we shall show a very interesting result on $\mu$-semi-stable vector

Received August 7, 1978. 
bundles on an algebraic surface (Theorem 4.6) whose proof is almost a modification of some parts of $\S 4$ and $\S 5$ of an excellent paper by Barth ([2]). Theorem 4.6 plays an important role in $\S 5$.

The author believes that one of the best ways to prove the boundedness is to show the stronger statement $B_{n, r}^{\prime}(\Lambda)$. If one reads the proof of Proposition 5.6 carefully, he may agree with the author. From Proposition 5.6, we can deduce easily that $B_{n, 3}^{\prime}(\Lambda)$ and $B_{n, 4}^{\prime}(\Lambda)$ hold for all $n$ if $\Lambda$ is a field of characteristic zero (Theorem 5.7). Unfortunately, Theorem 4.6 is false if the characteristic of the base field is positive. Thus, so long as the proof of Proposition 5.6 depends on Theorem 4.6, we are not able to remove the restriction on $\Lambda$ from Theorem 5.7. The author found a weaker result was enough to prove Proposition 5.6, (2) and hence $B_{n, 3}^{\prime}(\Lambda)$ holds and it is going to be written elsewhere (see Remark 5.8).

Throughout this paper, a key role is played by the Harder-Narasimhan filtration of a torsion free coherent sheaf (Definition 1.3). The author wishes to express his hearty thanks to Professor M. S. Narasimhan who pointed out the notion of the filtration to the author when he came to Kyoto in November, 1976.

\section{§1. Harder-Narasimhan filtration}

Throughout this section, we shall fix an arbitrary field $k$ and $\bar{k}$ denotes an algebraic closure of $k$. Let $X$ be a non-singular projective variety over $k$ (i.e., a smooth, projective, geometrically integral scheme over $k$ ) and $\mathcal{O}_{X}(1)$ a very ample invertible sheaf on $X$. For a coherent sheaf $E$ on $X_{K}=X \otimes_{k} K, d\left(E, \mathcal{O}_{X}(1)\right)$ or abbreviately $d(E)$ denotes the degree of the first Chern class $c_{1}(E)$ of $E$ with respect to $\mathcal{O}_{X_{K}}(1)=\mathcal{O}_{X}(1) \otimes_{k} K$, where $K$ is an extension field of $k$. We denote the rank of $E$ at the generic point of $X$ by $r(E)$. When $r(E) \neq 0, \mu(E)$ is defined as follows;

$$
\mu(E)=d\left(E, \mathcal{O}_{X}(1)\right) / r(E) .
$$

Note that for every extension field $L$ of $K, \mu(E)=\mu\left(E \otimes_{K} L\right)$.

Definition 1.1. Let $E$ be a coherent sheaf on $X$. $E$ is $\mu$-semi-stable if (1) $E$ is torsion free and (2) for every coherent subsheaf $F$ of $E_{\vec{k}}=E$ $\otimes_{k} \bar{k}$ with $F \neq 0, \mu(F) \leq \mu(E)$.

Remark 1.2. $E$ is torsion free if and only if so is $E_{\vec{k}}$ because $X$ is geometrically integral. 
The following notion is due to Harder and Narasimhan ([5]).

Definition 1.3. Let $E$ be a torsion free coherent sheaf on $X$. A Harder-Narasimhan filtration of $E$ is a filtration $0=E_{0} \subsetneq E_{1} \subsetneq \cdots \subsetneq E_{\alpha}$ $=E$ with the following properties;

(a) $E_{i} / E_{i-1}$ is $\mu$-semi-stable for $1 \leq i \leq \alpha$,

(b) $\mu\left(E_{i} / E_{i-1}\right)>\mu\left(E_{i+1} / E_{i}\right)$ for $1 \leq i \leq \alpha-1$.

Remark 1.4. It is easy to see that if $0=E_{0} \subsetneq E_{1} \subsetneq \cdots \subsetneq E_{\alpha}=E$ is a Harder-Narasimhan filtration, then so is $0=E_{0} \otimes_{k} L \subsetneq E_{1} \otimes_{k} L \subsetneq \cdots$ $\subsetneq E_{\alpha} \otimes_{k} L=E \otimes_{k} L$ for every extension field $L$ of $k$.

The existence and the uniqueness of a Harder-Narasimhan filtration are proved in [5] when $X$ is a curve and $k=\bar{k}$. If $k=\bar{k}$, one can easily generalize their proof to arbitrary dimensions (see [12]). The results hold good without assuming $k=\bar{k}$. In fact,

Proposition 1.5. Every torsion free coherent sheaf $E$ on $X$ has a unique Harder-Narasimhan filtration.

Proof. The uniqueness is easily deduced from Remark 1.4 and the uniqueness in the case of $k=\vec{k}$. For a proof of existence, take a HarderNarasimhan filtration $0=\bar{E}_{0} \subsetneq \bar{E}_{1} \subsetneq \cdots \subsetneq \bar{E}_{\alpha}=\bar{E}=E \otimes_{k} \bar{k}$. Let $\phi$ be a homomorphism of $\bar{E}_{1}$ to $\bar{E} / \bar{E}_{1}$ and $\beta$ the integer such that $\operatorname{im}(\phi) \subset \bar{E}_{\beta} / \bar{E}_{1}$ and $\operatorname{im}(\phi) \nless \bar{E}_{\beta-1} / \bar{E}_{1}$. If $\beta>1$, then we have a non-zero homomorphism $\bar{\phi}$ of $\bar{E}_{1}$ to $\bar{E}_{\beta} / \bar{E}_{\beta-1}$. This is not the case because $\mu\left(\bar{E}_{1}\right)>\mu\left(\bar{E}_{\beta} / \bar{E}_{\beta-1}\right)$ and both $\bar{E}_{1}$ and $\bar{E}_{\beta} / \bar{E}_{\beta-1}$ are $\mu$-semi-stable. Therefore, $\beta=1$ and $\phi=0$, and hence $\operatorname{Hom}_{\mathscr{O}_{\bar{X}}}\left(\bar{E}_{1}, \bar{E} / \bar{E}_{1}\right)=0$.

Let us consider the Quot-scheme $Q$ of $E / X / k$. Let $\bar{x}$ be the $\bar{k}$-valued geometric point of $Q$ corresponding to $\bar{E} \rightarrow \bar{E} / \bar{E}_{1} \rightarrow 0$ and let $x$ be the scheme point which is the image of $\bar{x}$. The residue field $K$ of $\mathcal{O}_{Q, x}$ is a finite algebraic extension of $k$. Then, $\bar{E}_{1}=E_{1} \otimes_{K} \bar{k}$ for a coherent sheaf on $E_{1}$ on $X_{K}$. If $K$ is not purely inseparable over $k$, then it contradicts the uniqueness of $\bar{E}_{1}$ (see Remark 1.6 below). For the maximal ideal $\mathfrak{m}$ of $\left(\mathcal{O}_{Q, x}\right) \otimes_{k} K, \mathrm{~m} / \mathrm{m}^{2} \otimes_{K} \bar{k}=\operatorname{Hom}_{O_{\bar{X}}}\left(\overline{E_{1}}, \bar{E} / \bar{E}_{1}\right)^{\vee}=0$ (see [4]), and hence $\mathfrak{m}=0$ by Nakayama's lemma. Therefore, $\left(\mathcal{O}_{Q, x}\right) \otimes_{k} K$ is a field, which means that $\mathcal{O}_{Q, x}=K=k$. By induction on the length of the filtration, we have our assertion.

q.e.d.

Remark 1.6. Let $E$ be a torsion free coherent sheaf on $X$ and $0=$ 
$E_{0} \subsetneq E_{1} \subsetneq \cdots \subsetneq E_{\alpha}=E$ the Harder-Narasimhan filtration. Set $m_{i}=$ $\max \left\{\mu(F) \mid F\right.$ : coherent subsheaf of $\left.E / E_{i}\right\}$ and $H=\{F \mid F$ : coherent subsheaf of $E / E_{i}$ with $\left.\mu(F)=m_{i}\right\}$. Then $E_{i+1} / E_{i}$ is a unique maximal member of $H$ (see [5], the proof of Lemma 1.3.6 and Lemma 1.3.7). Thus, for a coherent subsheaf $F$ of $E / E_{i}$, if $\mu(F)=\mu\left(E_{i+1} / E_{i}\right), r(F)=r\left(E_{i+1} / E_{i}\right)$ and if $\left(E / E_{i}\right) / F$ is torsion free, then $F=E_{i+1} / E_{i}$.

\section{§2. Construction of a subsheaf}

In this section, $k$ is assumed to be algebraically closed and the couple $\left(X, \mathcal{O}_{X}(1)\right)$ is the same as in the preceding section. For a vector subspace $V$ of $H^{0}\left(X, \mathcal{O}_{X}(1)\right)$, let $L$ be the linear subsystem of the complete linear system $\left|\mathcal{O}_{X}(1)\right|$ defined by $V$. Assume that $L$ is very ample. Set $P=P\left(V^{\vee}\right)$ and $G=\operatorname{Grass}_{2}\left(V^{\vee}\right)$, the Grassmann variety of 2-dimensional vector subspaces of $V$, where $V^{\vee}$ is the dual vector space of $V$. $P$ parametrizes all the divisors in $L$ and a non-empty open subset $G_{0}$ of $G$ does all the subschemes of codimension 2 in $X$ which are complete intersections of two members of $L$. Let $T \subset P \times{ }_{k} G$ be the flag variety which defines the incidence correspondence between $P$ and $G$. Set $T_{0}=T \times{ }_{G} G_{0}$ and let $\pi_{1}$ (or, $\pi_{2}$ ) be the projection of $T_{0}$ to $P$ (or, $T_{0}$ to $G_{0}$, resp.). On $T_{0}$, there is an effective Cartier divisor $\tilde{X}$ of $X \times{ }_{k} T_{0}$ such that for a point $t \in T_{0}, \tilde{X}_{t}$ is the divisor in $L \otimes_{k} k(t)$ corresponding to $\pi_{1}(t) \in P$. Moreover, we are able to construct an effective Cartier divisor $\tilde{Y}$ of $\tilde{X}$ such that for a point $t \in T_{0}, \tilde{Y}_{t}$ is the subscheme of $X \otimes_{k} k(t)$ defined by the point $\pi_{2}(t) \in G_{0}$. Note that both $\tilde{X}$ and $\tilde{Y}$ are flat over $T_{0}$.

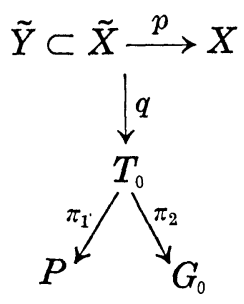

Now, for a point $u$ of $G_{0}, \pi_{2}^{-1}(u)=\boldsymbol{P}_{k(u)}^{1}$ and $\pi_{1}$ induces a linear embedding of $\boldsymbol{P}_{k(u)}^{1}$ to $P$. The subscheme of $X$ corresponding to $u$ is the base locus of the linear pencil in $L \otimes_{k} k(u)$ defined by $\pi_{1}\left(\boldsymbol{P}_{k(u)}^{1}\right)$. From this view point, $G_{0}$ can be regarded as a parametrizing space of linear pencils in $L$ which have base loci of codimension 2 .

$U_{0}$ denotes the maximal open set of $T_{0}$ over which both $\tilde{X}$ and $\tilde{Y}$ are 
smooth. Then $U_{0}$ is a non-empty open set of $T_{0}$. Let $E$ be a coherent torsion free sheaf on $X$ and $\tilde{E}=p_{U_{0}}^{*}(E)$. Shrinking $U_{0}$ if necessary, we may assume that $\tilde{E}$ is flat over $U_{0}$ and for every geometric point $t$ of $U_{0}$, $\tilde{E} \otimes k(t)$ is torsion free (see [8] Proposition 2.1 and [9] Lemma 1.6). Let $u$ be the generic point of $U_{0}$ and $F=E \otimes k(u)$. Take the HarderNarasimhan filtration of $F$

$$
0=F_{0} \subsetneq F_{1} \subsetneq \cdots \sqsubseteq F_{\alpha}=F .
$$

By Proposition 1.5, the above filtration is defined over $k(u)$. Thus the quotient coherent sheaf $F / F_{1}$ of $F$ defines a morphism $s_{0}$ of $\operatorname{Spec}(k(u))$ to $Q$, where $Q$ is the $Q$ uot-scheme of $\tilde{E} / \tilde{X}_{U_{0}} / U_{0}$. Since $U_{0}$ is an integral scheme, there are a non-empty open set $U_{1}$ of $U_{0}$ and a morphism $s$ of $U_{1}$ to $Q$ with $\pi s=\mathrm{id}_{U_{1}}$ and $s \otimes k(u)=s_{0}$, where $\pi$ is the structure morphism of $Q$ as a $U_{0}$-scheme. Let $\tilde{E}_{U_{1}} \stackrel{\phi_{1}}{\longrightarrow} \tilde{E}_{1}^{\prime} \longrightarrow 0$ be the pull-back of the universal quotient sheaf on $X_{Q}$ by $s$. Applying the above argument to $\tilde{E}_{1}^{\prime}$ and $F_{2} / F_{1}$, we obtain a non-empty open set $U_{2}$ in $U_{1}$ and a surjective homomorphism $\left(\tilde{E}_{1}^{\prime}\right)_{U_{2}} \stackrel{\phi_{2}}{\longrightarrow} \tilde{E}_{2}^{\prime}$ of $U_{2}$-flat coherent sheaves on $\tilde{X}_{U_{2}}$ such that $\phi_{2} \otimes k(u)$ is the quotient homomorphism $F / F_{1} \rightarrow F / F_{2}$. Repeating these procedures, we have a non-empty open set $U_{\alpha}$ of $U_{0}$ and surjective homomorphisms $\phi_{i}: \tilde{E}_{i}^{\prime} \rightarrow \tilde{E}_{i+1}^{\prime}(0 \leq i \leq \alpha-1)$ of $U_{\alpha}$-flat, coherent sheaves on $\tilde{X}_{U_{\alpha}}$ such that $\tilde{E}_{0}^{\prime}=\tilde{E}_{U_{\alpha}}$ and $\phi_{i} \otimes k(u)$ is the quotient homomorphism $F / F_{i} \rightarrow F / F_{i+1}$. Set $\tilde{E}_{i}=\operatorname{ker}\left(\phi_{i-1} \cdots \phi_{0}\right)$ and $\tilde{E}_{0}=0$, then each $\tilde{E}_{i}$ is $U_{\alpha}$-flat and $\tilde{E}_{i} \otimes k(u)=F_{i}$. In this situation, we obtain a non-empty open subset $U^{\prime}$ of $U_{\alpha}$ such that for all points $t$ of $U^{\prime}$, evey $\left(\tilde{E}_{i} / \tilde{E}_{i-1}\right) \otimes k(t)$ is $\mu$-semistable ([8] Theorem 2.8). Clearly, for all points $t$ of $U^{\prime}, \mu\left(\left(\tilde{E}_{i} / \tilde{E}_{i-1}\right) \otimes k(t)\right)$ $=\mu\left(F_{i} / F_{i-1}\right)$ and hence,

$$
0=\tilde{E}_{0} \otimes k(t) \subsetneq \tilde{E}_{1} \otimes k(t) \subsetneq \cdots \subsetneq \tilde{E}_{\alpha} \otimes k(t)=\tilde{E} \otimes k(t)
$$

is the Harder-Narasimhan filtration of $E \otimes k(t)$.

For the generic point $u$ of $U^{\prime},\left.F_{i}\right|_{\tilde{Y}_{u}}$ is a torsion free sheaf ([9] Lemma 1.6), whence $\left.F_{i}\right|_{\tilde{Y}_{u}}$ is a subsheaf of $\left.F\right|_{\tilde{Y}_{u}}$. Thus there exists a non-empty open subset $U$ of $U^{\prime}$ such that each $j^{*}\left(\left(\tilde{E}_{i}\right)_{U}\right)=\tilde{J}_{i}$ is a subsheaf of $\tilde{J}=$ $j^{*}\left(\left(\tilde{E}_{\alpha}\right)_{U}\right)$ and that each $\tilde{J} / \tilde{J}_{i}$ is $U$-flat for $0 \leq i \leq \alpha$, where $j: \tilde{Y}_{U} \rightarrow \tilde{X}_{U}$ is the closed immersion. Then we can find an open subset $W_{0}$ of $U$, which may be empty, such that $W_{0}=\left\{t \in U \mid\left(\tilde{J}_{i} / \tilde{J}_{i-1}\right) \otimes k(t)\right.$ are $\mu$-semi-stable, $1 \leq i \leq \alpha\}$. Since

$$
\mu\left(\left(\tilde{J}_{i} / \tilde{J}_{i-1}\right) \otimes k(t)\right)=\mu\left(\left(\tilde{E}_{i} / \tilde{E}_{i-1}\right) \otimes k(t)\right)
$$


for all points $t$ of $W_{0}$,

$$
0=\tilde{J}_{0} \otimes k(t) \sqsubseteq \tilde{J}_{1} \otimes k(t) \subsetneq \cdots \sqsubseteq \tilde{J}_{\alpha} \otimes k(t)=\left.\tilde{E}\right|_{\tilde{r}_{t}}
$$

is the Harder-Narasimhan filtration of $\left.\tilde{E}\right|_{\tilde{Y}_{t}}$.

On the other hand, by the same procedure as the construction of $\left\{\tilde{E}_{i}\right\}$, we obtain a filtration $0=\tilde{H}_{0} \subsetneq \tilde{H}_{1} \subsetneq \cdots \sqsubseteq \tilde{H}_{\beta}=\tilde{J}_{W}$ on a non-empty open set $W$ of $U$ such that each $\tilde{H}_{\beta} / \tilde{H}_{i}$ is $W$-flat and that for every point $t$ of $W$,

$$
0=\tilde{H}_{0} \otimes k(t) \sqsubseteq \tilde{H}_{1} \otimes k(t) \sqsubseteq \cdots \sqsubseteq \tilde{H}_{\beta} \otimes k(t)=\left.\tilde{E}\right|_{\tilde{Y}_{t}}
$$

is the Harder-Narasimhan filtration of $\left.\tilde{E}\right|_{\tilde{I}_{t}}$. Set $\ell_{i}=\min \left\{\ell \mid\left(\tilde{J}_{i}\right)_{W} \rightarrow \tilde{J}_{W} / \tilde{H}_{\ell}\right.$ is zero on the generic fibre of $\tilde{Y}\}$. Then there is a non-empty open set $W_{i}^{\prime}$ of $W$ such that $\left(\tilde{J}_{i}\right)_{W_{i}^{\prime}}$ is contained in $\left(\tilde{H}_{\ell_{i}}\right)_{W_{i}^{\prime}}$. We let $C_{i}=\operatorname{Supp}\left(\operatorname{coker}\left(\left(\tilde{J}_{i}\right)_{W_{i}^{\prime}}\right.\right.$ $\left.\left.\rightarrow\left(\tilde{H}_{\ell_{i}}\right)_{W_{i}^{\prime}}\right)\right)$. Since $q\left(C_{i}\right)$ is a closed set in $W_{i}^{\prime}, W_{i}=W_{i}^{\prime}-q\left(C_{i}\right)$ is an open subset of $T_{0}$. On $W_{i}, \tilde{J}_{i}$ coincides with $\tilde{H}_{\ell_{i}}$. Furthermore, $W_{i}$ is nonempty if and only if, for the generic point $u$ of $T_{0}, \tilde{J}_{i} \otimes k(u)=\left.F_{i}\right|_{\tilde{Y}_{u}}$ is a filter of the Harder-Narasimhan filtration of $\left.\tilde{E}\right|_{\tilde{Y}_{u}}$.

Summerizing the above results, we have

Lemma 2.2. Let $E$ be a torsion free coherent sheaf on $X$.

1) There exists a non-empty open set $U$ of $T_{0}$ (see diagram (2.1)) such that $\tilde{E}=p^{*}(E)_{U}$ has a filtration $\Phi: 0=\tilde{E}_{0} \subseteq \tilde{E}_{1} \subseteq \cdots \sqsubseteq \tilde{E}_{\alpha}=\tilde{E}$ with the following properties (a), (b), (c) and (d);

(a) $\tilde{X}_{U}$ and $\tilde{Y}_{U}$ is smooth over $U$,

(b) every $\tilde{E} / \tilde{E}_{i}$ is flat over $U(1 \leq i \leq \alpha)$,

(c) for every point $t$ of $U$,

$$
\Phi \otimes k(t): 0=\tilde{E}_{0} \otimes k(t) \subsetneq \tilde{E}_{1} \otimes k(t) \subsetneq \cdots \subsetneq \tilde{E}_{\alpha} \otimes k(t)=\left.E\right|_{\tilde{X}_{t}}
$$

is the Harder-Narasimhan filtration of $\left.E\right|_{\tilde{X}_{t}}$,

(d) for the closed immersion $j: \tilde{Y}_{U} \rightarrow \tilde{X}_{U}$, each $\tilde{J}_{i}=j^{*}\left(\tilde{E}_{i}\right)$ is a subsheaf of $\tilde{J}=j^{*}(\tilde{E})$ and each $\tilde{J} / \tilde{J}_{i}$ is flat over $U(1 \leq i \leq \alpha)$.

2) There exists an open set $W_{0}$ of $U$ such that for every point $t$ of $W_{0}$, the filtration $\left.\Phi\right|_{\tilde{r}_{t}}$ has the following property $\left(\mathrm{e}_{0}\right)$ and that $W_{0}$ is nonempty if and only if $\left.\Phi\right|_{\tilde{Y}_{t}}$ has the property $\left(\mathrm{e}_{0}\right)$ when $t$ is the generic point of $U$;

(e $\left.\mathrm{e}_{0}\right)\left.\Phi\right|_{\tilde{Y}_{t}}$ is the Harder-Narasimhan filtration of $\tilde{J} \otimes k(t)$.

3) There exists an open set $W_{i}(1 \leq i<\alpha)$ of $U$ such that for every 
point $t$ of $W_{i}, \tilde{J}_{i} \otimes k(t)$ has the following property $\left(\mathrm{e}_{i}\right)$ and that $W_{i}$ is nonempty if and only if $\tilde{J}_{i} \otimes k(t)$ has the property $\left(\mathrm{e}_{i}\right)$ when $t$ is the generic point of $U$;

$\left(\mathrm{e}_{i}\right) \quad \tilde{J}_{i} \otimes k(t)$ is a filter of the Harder-Narasimhan filtration of $\tilde{J} \otimes k(t)$.

Remark 2.3. 1) Let $f: X \rightarrow S$ be a smooth, projective, geometrically integral morphism of noetherian schemes, $\mathcal{O}_{X}(1)$ an $f$-very ample invertible sheaf on $X$ and $E$ a coherent sheaf on $X$. Assume that $S$ is irreducible and reduced. Let $F_{1}$ and $F_{2}$ be coherent subsheaves of $E$ with the following properties;

(a) $E / F_{1}$ and $E / F_{2}$ are flat over $S$,

(b) $r\left(F_{1}\right)=r\left(F_{2}\right)$,

(c) for the generic point $s$ of $S, E \otimes k(s)$ is torsion free and both $F_{1} \otimes k(s)$ and $F_{2} \otimes k(s)$ are filters of the Harder-Narasimhan filtration of $E \otimes k(s)$. Then $F_{1}=F_{2}$.

2) By virtue of 1), the filter $\Phi$ in 1) of Lemma 2.2 is unique.

Proof of 1). Let $Q$ be the Quot-scheme of $E / X / S$. By (a), $E / F_{i}$ defines a section $g_{i}$ of $S$ to $Q$. By virtue of (b), (c) and the uniqueness of the Harder-Narasimhan filtration, $g_{1, s}=g_{2, s}$ as morphisms of $\operatorname{Spec}(k(s)$ ) to $Q_{s}$. Since $Q$ is separated over $S$ and since $S$ is irreducible and reduced, $g_{1}=g_{2}$.

q.e.d.

As an application of the above, we have the following which plays a key role in the sequal.

Proposition 2.4. Let $E$ be a torsion free, coherent sheaf on $X$ and let $L$ be a very ample linear subsystem of $\left|\mathcal{O}_{X}(1)\right|$. Assume that $\operatorname{dim} X \geq 3$ and $W_{i}$ for $E$ in Lemma 2.2 is not empty for some $0<i<\alpha$. Then there is a coherent subsheaf $E_{i}$ of $E$ such that $\mu\left(E_{i}\right)=\mu\left(\tilde{E}_{i} \otimes k(t)\right)$ and $r\left(E_{i}\right)=$ $r\left(\tilde{E}_{i} \otimes k(t)\right)$ for a point $t$ of $W_{i}$.

Proof. Since $\pi_{2}: T_{0} \rightarrow G_{0}$ is flat, $\pi_{2}\left(W_{i}\right)$ is a non-empty open set of $G_{0}$. Pick a $k$-rational point $v$ of $\pi_{2}\left(W_{i}\right)$. Since the base locus $Y$ of the linear pencil $\pi_{2}^{-1}(v)$ is smooth and since $L$ is very ample, we see that for every $t \in \pi_{2}^{-1}(v)$, the singular locus of $\tilde{X}_{t}$ is at most a finite set of points. This and the assumption that $\operatorname{dim} X \geq 3$ imply that for every $t \in \pi_{2}^{-1}(v), \tilde{X}_{t}$ is reduced and irreducible. Moreover, $f: Z=\tilde{X} \times_{T_{0}} \pi_{2}^{-1}(v) \rightarrow X$ is the blowing-up of $X$ with center $Y, D=Y \times_{T_{0}} \pi_{2}^{-1}(v)$ is the exceptional divisor of $f$ and $D \cong Y \times_{k} \pi_{2}^{-1}(v)$. 


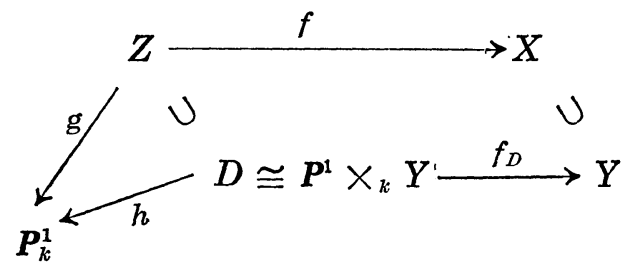

For each point $t$ of $P_{k}^{1}, Z_{t}=g^{-1}(t)$ is the member of the linear pencil $\pi_{2}^{-1}(v)$ corresponding to $t$ and $h^{-1}(t)=D_{t} \cong Y$ as subschemes of $Z_{t} \subset X$. Set $V=W_{i} \cap \pi_{2}^{-1}(v)$. Then $\tilde{E}_{V}=\tilde{E} \otimes_{\mathcal{O W}_{i}} \mathcal{O}_{V}$ and $\tilde{E}_{i, V}=\tilde{E}_{i} \otimes_{\mathcal{O W}_{i}} \mathcal{O}_{V}$ are flat over $V$ and this is a subsheaf of that because of the property (b) of Lemma 2.2. Let $B$ be the subsheaf of torsions of $f^{*}(E)$ and let $E^{\prime}=f^{*}(E) / B$. Then $E^{\prime}$ is $g$-flat and clearly $E_{V}^{\prime} \cong \tilde{E}_{V}$. It is easy to construct a coherent subsheaf $E_{i}^{\prime}$ of $E^{\prime}$ such that $F^{\prime}=E^{\prime} / E_{i}^{\prime}$ is g-flat and $F_{V}^{\prime} \cong \tilde{E}_{V} / \tilde{E}_{i, V}$ as quotient sheaves of $E_{V}^{\prime}$. Note that $f_{D}^{*}\left(\left.E\right|_{Y}\right)=\left.E^{\prime}\right|_{D}$.

Pick a point $t$ of $\boldsymbol{P}_{k}^{1}-V$ and let $N$ be the torsion part of $F_{t}^{\prime} F^{\prime} \otimes k(t)$ (note that $Z_{t}$ is a projective variety with at most a finite number of singular points). Suppose that $\operatorname{codim}\left(\operatorname{Supp}(N), Z_{t}\right)=1$, then $\chi(N(m))=$ $a m^{n-1} /(n-1) !+$ terms of degree $<n-1$ with $a$ some positive integer, where $\operatorname{dim} X=n+1$. For $F_{0}^{\prime}=F^{\prime} \otimes k\left(t_{0}\right), t_{0} \in V$,

$$
\begin{aligned}
\chi\left(F_{t}^{\prime}(m)\right)= & \chi\left(F_{0}^{\prime}(m)\right)=r\left(F_{0}^{\prime}\right) d m^{n} / n !+\left(d\left(F_{0}^{\prime}\right)+r\left(F_{0}^{\prime}\right) \kappa / 2\right) m^{n-1} /(n-1) ! \\
& + \text { terms of degree }<n-1,
\end{aligned}
$$

where $\kappa$ is the degree of a canonical divisor of $Z_{t_{0}}$ and $d$ is the degree of $X$ with respect to $\mathcal{O}_{X}(1)$. Thus, for $\bar{F}^{\prime}=F_{t}^{\prime} / N$,

$$
\begin{aligned}
\chi\left(\bar{F}^{\prime}(m)\right)= & r\left(F_{0}^{\prime}\right) d m^{n} / n !+\left(d\left(F_{0}^{\prime}\right)-a+r\left(F_{0}^{\prime}\right) \kappa / 2\right) m^{n-1} /(n-1) ! \\
& + \text { terms of degree }<n-1 .
\end{aligned}
$$

Let us consider the sheaf $H=\left.\bar{F}^{\prime}\right|_{D_{t}}$. Since $\bar{F}^{\prime}$ is torsion free, we obtain the following exact sequence;

$$
0 \longrightarrow \bar{F}^{\prime}(-1) \longrightarrow \bar{F}^{\prime} \longrightarrow H \longrightarrow 0 \text {. }
$$

Thus we have

$$
\begin{aligned}
& \chi(H(m))=\chi\left(\bar{F}^{\prime}(m)\right)-\chi\left(\bar{F}^{\prime}(m-1)\right)=r(H) d m^{n-1} /(n-1) !+\left(d\left(F_{0}^{\prime}\right)-a\right. \\
& \left.\quad+r(H) \kappa^{\prime} / 2\right) m^{n-2} /(n-2) !+\text { terms of degree } n-2 \\
& \text { and } r(H)=r\left(F_{0}^{\prime}\right),
\end{aligned}
$$

where $\kappa^{\prime}$ is the degree of a canonical divisor of $D_{t} \cong Y$. Therefore, 


$$
\mu(H)=\left(d\left(F_{0}^{\prime}\right)-a\right) / r(H)<d\left(F_{0}^{\prime}\right) / r\left(F_{0}^{\prime}\right)=\mu\left(F_{0}^{\prime}\right) .
$$

On the other hand, $\left.E^{\prime}\right|_{D_{t}} \cong j_{t}^{*}\left(\left.E\right|_{Y}\right)$ and $H$ is a quotient sheaf of $\left.E^{\prime}\right|_{D_{t}}$ with $r(H)=r\left(F_{0}^{\prime}\right)=r\left(\left.F_{0}^{\prime}\right|_{D_{t}}\right)$, where $j_{t}$ is the isomorphism of $D_{t}$ to $Y$. These and Theorem 2 of [12] implies that $\mu(H) \geq \mu\left(\left.F_{0}^{\prime}\right|_{D_{t_{0}}}\right)=\mu\left(F_{0}^{\prime}\right)$. This is a contradiction, whence codim $\left(\operatorname{Supp}(N), Z_{t}\right) \geq 2$. Therefore, if $C$ is the set of pinch points of $F^{\prime}$, i.e., the set of points at which $F^{\prime}$ is not locally free, then

(2.4.1) $\operatorname{codim}\left(C_{t}, Z_{t}\right) \geq 2$ and hence, $C_{t} \not \supset D_{t}$ for all $t \in \boldsymbol{P}_{k}^{1}$.

Let $0=H_{0} \subsetneq H_{1} \subseteq \cdots \subseteq H_{\beta}=\left.E\right|_{Y}$ be the Harder-Narasimhan filtration of $\left.E\right|_{Y}$ and let $\tilde{H}_{j}=f_{D}^{*}\left(H_{j}\right)$. Let $K$ be the torsion part of $\left.F^{\prime}\right|_{D}$ and let $I$ be $\left(\left.F^{\prime}\right|_{D}\right) / K$. Then, we see that $\operatorname{Supp}(K) \subseteq C \cap D, I$ is flat over $P_{k}^{1}$ and $I_{V}=\left.F^{\prime}\right|_{D_{V}}$. Moreover, $I$ coincides with $\left(\left.E^{\prime}\right|_{D}\right) / \tilde{H}_{\ell}$ for an $\ell$ by virtue of the property $\left(\mathrm{e}_{i}\right)$ for $W_{i}$ and Remark 2.3, (1). Thus $C_{t} \cap D_{t}$ is $j_{t}^{-1}(A)$ if $t \in V$, where $A$ is the set of pinch points of $H_{\ell}$. From this we infer that $f_{D}(C \cap D)=A \cup\left(\bigcup_{t \in P_{k}^{1}-V} j_{t}\left(C_{t} \cap D_{t}\right)\right)$. Since $\boldsymbol{P}_{k}^{1}-V$ is a finite set, (2.4.1) shows that $f_{D}(C \cap D) \neq Y$. We have therefore

(2.4.2) On $D-(C \cap D),\left.F^{\prime}\right|_{D}$ is isomorphic to $f_{D}^{*}\left(\left(\left.E\right|_{Y}\right) / H_{\ell}\right)$ as quotient sheaf of $\left.E^{\prime}\right|_{D}$ and $f_{D}(C \cap D) \neq Y$.

By replacing $E$ by $E(m), m \gg 0$, we may assume that $E$ is generated by its global sections:

$$
\psi: \stackrel{\mathcal{O}_{x}^{M}}{\longrightarrow} \longrightarrow E \longrightarrow 0 \text {. }
$$

Pulling back $\psi$ to $Z$ and composing it with the homomorphism $f^{*}(E) \rightarrow E^{\prime}$, we have surjective homomorphisms $\tau_{1}$ and $\tau_{2}$;

$$
\mathcal{O} \oplus \stackrel{\oplus_{Z}^{M}}{\longrightarrow} E^{\prime} \stackrel{\tau_{2}}{\longrightarrow} F^{\prime} \text {. }
$$

Let $C^{\prime}$ be the set of pinch points of $E$. Then, for general points $t$ of $\boldsymbol{P}_{k}^{1}, \operatorname{codim}\left(f^{-1}\left(C^{\prime}\right) \cap Z_{t}, Z_{t}\right) \geq 2$ and $\operatorname{codim}\left(C^{\prime} \cap Y, Y\right) \geq 2$. Set $Z_{0}=Z-$ $f^{-1}\left(f(C) \cup C^{\prime}\right), \quad X_{0}=X-f(C) \cup C^{\prime}$ and $f_{0}=\left.f\right|_{Z_{0}}$. The restrictions of $\tau_{1}$ and $\tau_{2}$ to $Z_{0}$ define a morphism $v: Z_{0} \rightarrow$ Flag $\left(M, r\left(E^{\prime}\right), r\left(F^{\prime}\right)\right)$; the flag variety of pairs of quotient vector spaces $\left(V_{1}, V_{2}\right)$ of an $M$-dimensional vector space such that $\operatorname{dim} V_{1}=r\left(E^{\prime}\right), \operatorname{dim} V_{2}=r\left(F^{\prime}\right)$ and $V_{2}$ is a quotient vector space of $V_{1}$. By (2.4.2), $f_{D}^{*}\left(\left(\left.E\right|_{Y}\right) / H_{\ell}\right)=\left.F^{\prime}\right|_{D}$ on $Z_{0} \cap D$ as quotient bundles of $\left.E^{\prime}\right|_{Z_{0} \cap D}$ and hence, $v\left(f^{-1}(y)\right)$ is one point for all $y \in Y \cap X_{0}$. Thus $v$ factors through $X_{0}$, that is, $v=v^{\prime} f_{0}$ for a $v^{\prime}: X_{0} \rightarrow$ Flag $\left(M, r\left(E^{\prime}\right), r\left(F^{\prime}\right)\right)$. 
This means that there are locally free quotient coherent sheaves $\eta_{1}: \mathcal{O}_{X_{0}}^{\oplus M}$ $\rightarrow E_{0}$ and $\eta_{2}: E_{0} \rightarrow F_{0}$ such that $\left.f_{0}^{*}\left(E_{0}\right) \cong E^{\prime}\right|_{z_{0}} \cong f_{0}^{*}\left(\left.E\right|_{X_{0}}\right),\left.f_{0}^{*}\left(F_{0}\right) \cong F^{\prime}\right|_{Z_{0}}$, $f_{0}^{*}\left(\eta_{1}\right) \cong \tau_{1}$ and $f_{0}^{*}\left(\eta_{2}\right) \cong \tau_{2}$. Since $\left.E_{0} \cong f_{0^{*}} f_{0}^{*}\left(E_{0}\right) \cong f_{0^{*}} f_{0}^{*}\left(\left.E\right|_{X_{0}}\right) \cong E\right|_{X_{0}}, \eta_{2}$ induces a surjective homomorphism of $\left.E\right|_{x_{0}}$ to $F_{0}$. Then we can extend this to a surjective

$$
\zeta: E \rightarrow F \quad \text { with } F \text { torsion free . }
$$

Set $E_{i}=\operatorname{ker}(\zeta)$, then $E_{i}$ meets our requirement. In fact, for general $t \in \boldsymbol{P}_{k}^{1}$, identify $Z_{t}$ by $f\left(Z_{t}\right) .\left.\quad F\right|_{\left(x_{0} \cap Z_{t}\right)}=\left.F_{0}\right|_{\left(X_{0} \cap Z_{t}\right)}=\left.F^{\prime}\right|_{Z_{0}, t}$ and from (2.4.1) and (2.4.2) we can deduce $\operatorname{codim}\left(Z_{t}-X_{0} \cap Z_{t}, Z_{t}\right) \geq 2$. Hence $d\left(\left.F\right|_{Z_{t}}\right)=$ $d\left(\left.F^{\prime}\right|_{z_{t}}\right)$ and $r\left(\left.F\right|_{z_{t}}\right)=r\left(\left.F^{\prime}\right|_{z_{t}}\right)$, which imply that $d\left(\left.\tilde{E}_{i}\right|_{z_{t}}\right)=d\left(\left.E_{i}\right|_{z_{t}}\right)$ and $r\left(\left.\tilde{E}_{i}\right|_{z_{t}}\right)=r\left(\left.E_{i}\right|_{z_{t}}\right)$. Therefore, $\mu\left(E_{i}\right)=\mu\left(\left.E_{i}\right|_{z_{t}}\right)=\mu\left(\tilde{E}_{i} \otimes k(t)\right)$ and $r\left(E_{i}\right)=$ $r\left(\left.E_{i}\right|_{z_{t}}\right)=r\left(\tilde{E}_{i} \otimes k(t)\right)$.

q.e.d.

\section{§3. Boundedness of semi-stable sheaves of rank 2}

In this section, we shall prove the boundedness of $\mu$-semi-stable sheaves of rank 2. In the first place, let us show the following interesting result which can be derived from Proposition 2.4 directly.

TheOREM 3.1. Let $X$ be a non-singular projective variety over an algebraically closed field $k$ and $\mathcal{O}_{X}(1)$ a very ample invertible sheaf on $X$. Let $E$ be a torsion free coherent sheaf on $X$ and $L$ a very ample linear subsystem of $\left|\mathcal{O}_{X}(1)\right|$. Assume that $\operatorname{dim} X \geq 2, r(E)<\operatorname{dim} X$ and that $E$ is $\mu$-semi-stable with respect to $\mathcal{O}_{X}(1)$. Then, for general members $Y$ of $L$, $\left.E\right|_{Y}$ is $\mu$-semi-stable with respect to $\mathcal{O}_{Y}(1)=\left.\mathcal{O}_{X}(1)\right|_{Y}$.

Proof. We shall prove our assertion by induction on $r(E)$. If $r(E)$ $=1$, then it is enough to show that $\left.E\right|_{Y}$ is torsion free and it follows from Lemma 1.6 of [9]. Assume that $r(E)>1$. Take the open set $U$ of $T_{0}$ and the filtration

$$
\Phi: 0=\tilde{E}_{0} \subsetneq \tilde{E}_{1} \subsetneq \cdots \subsetneq \tilde{E}_{\alpha}=\tilde{E}
$$

of Lemma 2.2. Pick a $k$-rational point $t$ in $U$. $\left.E\right|_{\tilde{x}_{t}}$ is not $\mu$-semi-stable if and only if $\alpha>1$. $\pi_{1}^{-1} \pi_{1}(t) \cap U$ is regarded as an open set of the linear system $\operatorname{Tr}_{\tilde{x}_{t}}(L)$. Now, assume that $\left.E\right|_{\tilde{x}_{t}}$ is not $\mu$-semi-stable. Then, $r\left(\left(\tilde{E}_{i} / \tilde{E}_{i-1}\right) \otimes k(t)\right)<r(E) \leq \operatorname{dim} \tilde{X}_{t}$. Thus, by our induction hypothesis, for general members $D$ of $\operatorname{Tr}_{\tilde{X}_{t}}(L)$, every $\left.\left(\tilde{E}_{i} / \tilde{E}_{i-1}\right) \otimes k(t)\right|_{D}$ is $\mu$-semi-stable. We may assume, therefore, that our filtration $\Phi$ of $\tilde{E}$ and open set $U$ 
have the property $\left(\mathrm{e}_{0}\right)$ of Lemma 2.2. Since $\operatorname{dim} X>r(E) \geq 2$, the above and Proposition 2.4 provide us with a coherent subsheaf $E_{1}$ of $E$ with $\mu\left(E_{1}\right)>\mu(E)$. This contradicts the assumption that $E$ is $\mu$-semi-stable. Hence, $\left.E\right|_{\tilde{x}_{t}}$ is $\mu$-semi-stable.

q.e.d.

Before showing the main result in this section, let us observe our problem from general view point and make it clear. Let $f: X \rightarrow S$ be a smooth, projective, geometrically integral morphism of noetherian schemes over a ring $\Lambda$ and let $\mathcal{O}_{X}(1)$ be an $f$-very ample invertible sheaf on $X$. Assume that the dimensions of fibres of $f$ are constant $n$. For a coherent sheaf $E$ on a fibre $X_{s}$ of $X$ over $S$, we can write

$$
\chi(E(m))=\sum_{i=0}^{n} a_{i}(E)\left(\begin{array}{c}
m+n-i \\
n-i
\end{array}\right)
$$

with some integers $a_{0}(E), \cdots, a_{n}(E)$, where $E(m)=E \otimes_{k(s)} \mathcal{O}_{X}(m)$.

For an integer $r$ and a sequence of integers $\left(a_{1}, \cdots, a_{n}\right), \Sigma_{X / S}\left(n, r, a_{1}\right.$, $\left.\cdots, a_{n}\right)$ is the family of the classes of coherent sheaves on the fibres of $X$ over $S$ such that $E$ on a fibre $X_{s}$ is contained in $\Sigma_{X / S}\left(n, r, a_{1}, \cdots, a_{n}\right)$ if and only if it has the following two properties:

(3.3.1) $E$ is $\mu$-semi-stable with respect to $\mathcal{O}_{X_{s}}(1)=\mathcal{O}_{X}(1) \otimes_{\mathcal{O}_{S}} k(s)$.

(3.3.2) $a_{0}(E)=r d, a_{1}(E)=a_{1}$ and $a_{i}(E) \geq a_{i}$ for $2 \leq i \leq n$, where $d$ is the degree of $X_{s}$ with respect to $\mathcal{O}_{X_{s}}(1)$.

The property (3.3.2) implies that, for $E \in \Sigma_{X / S}\left(n, r, a_{1}, \cdots, a_{n}\right), r(E)=$ $r$ and the degree of $E$ is constant on each connected component of $S$.

For integers $r, a_{1}$ and $a_{2}, \Sigma_{X / S}^{\prime}\left(n, r, a_{1}, a_{2}\right)$ is the family of the classes of coherent sheaves on the fibres of $X$ over $S$ such that $E$ on a fibre $X_{s}$ is contained in $\Sigma_{X / S}^{\prime}\left(n, r, a_{1}, a_{2}\right)$ if and only if $E$ has the property (3.3.1) and the following two properties:

(3.3.3) $E$ satisfies Serre's condition $\left(S_{2}\right)$ (EGA Ch. IV, 5.7.2).

(3.3.4) $a_{0}(E)=r d, a_{1}(E)=a_{1}$ and $a_{2}(E) \geq a_{2}$, where $d$ is the same as in (3.3.2).

Finally let us introduce another family. For a numerical polynomial $h(m)$ of degree $n, \Sigma_{X / S}^{\prime \prime}(n, r, h(m))$ is the family of the classes of coherent sheaves on the fibres of $X$ over $S$ such that $E$ is a member of $\Sigma_{X / S}^{\prime \prime}(n, r, h(m))$ if and only if $E$ has the property (3.3.1) and the following property: 


$$
r(E)=r \quad \text { and } \quad \chi(E(m))=h(m) .
$$

Let us consider the following statements:

(3.4.1) $B_{n, r}(\Lambda): \Sigma_{X / S}\left(n, r, a_{1}, \cdots, a_{n}\right)$ is bounded for all $f: X \rightarrow S, \mathcal{O}_{X}(1)$ and $\left(a_{1}, \cdots, a_{n}\right)$ whenever $n, r$ and $\Lambda$ are fixed.

(3.4.2) $B_{n, r}^{\prime}(\Lambda): \Sigma_{X / S}^{\prime}\left(n, r, a_{1}, a_{2}\right)$ is bounded for all $f: X \rightarrow S, \mathcal{O}_{X}(1)$ and $\left(a_{1}, a_{2}\right)$ whenever $n, r$ and $\Lambda$ are fixed.

(3.4.3) $B_{n, r}^{\prime \prime}(\Lambda): \Sigma_{X / S}^{\prime \prime}(n, r, h(m))$ is bounded for all $f: X \rightarrow S, \mathcal{O}_{X}(1)$ and $h(m)$ whenever $n, r$ and $\Lambda$ are fixed.

Lemma 3.5. (1) $B_{n, r}(\Lambda)$ implies $B_{n, r}^{\prime \prime}(\Lambda)$.

(2) $B_{n, r}^{\prime}(\Lambda)$ implies $B_{n, r}^{\prime \prime}(\Lambda)$.

Proof. (1) is trivial. Take a coherent sheaf $E$ on $X_{s}$ which is contained in $\Sigma_{X / S}^{\prime \prime}(n, r, h(m))$. We have only to show that $E$ is a $(b)$-sheaf for a sequence $(b)=\left(b_{0}, \cdots, b_{n}\right)$ of integers which depends only on the family $\Sigma_{X / S}^{\prime \prime}(n, r, h(m)$ ) (see [6] Théorème 1.13). Let $Y$ be the set of pinch points of $E$ and $i: X-Y \rightarrow X$ the natural immersion. Then $i_{*} i^{*}(E)=E^{\prime}$ is torsion free and coherent because $E$ is a subsheaf of a coherent, locally free sheaf $F$ and hence $E^{\prime}$ is a quasi-coherent subsheaf of $i_{*} i^{*}(F)$ $=F$. Moreover, $E^{\prime}$ satisfies the condition $\left(S_{2}\right)$ because $\operatorname{codim}(Y, X) \geq 2$ (EGA Ch. IV, 5.10.5). Consider the exact sequence

$$
0 \longrightarrow E \longrightarrow E^{\prime} \longrightarrow T \longrightarrow 0 \text {. }
$$

Since codim $(\operatorname{Supp}(T), X) \geq 2, \chi(T(m))=\mathrm{cm}^{n-2}+$ terms of degree $<n-2$, $c \geq 0$. Therefore, $E^{\prime}$ is a member of $\sum_{X / s}^{\prime}\left(n, r, a_{1}, a_{2}\right)$, where

$$
h(m)=r d\left(\begin{array}{c}
m+n \\
n
\end{array}\right)+\sum_{i=1}^{n} a_{i}\left(\begin{array}{c}
m+n-i \\
n-i
\end{array}\right) .
$$

$B_{n, r}^{\prime}(\Lambda)$ implies that there exists a sequence $(b)=\left(b_{0}, \cdots, b_{n}\right)$ of integers such that every member of $\Sigma_{x / S}^{\prime}\left(n, r, a_{1}, a_{2}\right)$ is a (b)-sheaf. Since for general members $s_{1}, \cdots, s_{n}$ of $H^{0}\left(X_{s}, \mathcal{O}_{X_{s}}(1)\right),\left.E\right|_{Y_{i}}$ is a subsheaf of $\left.E^{\prime}\right|_{Y_{i}}, E$ is also a $(b)$-sheaf, where $Y_{i}$ is the zero scheme of $s_{1}, \cdots, s_{i}$. q.e.d.

Let $p$ be the natural morphism of $D=\operatorname{Div}_{X / S}$ to $P=\operatorname{Pic}_{X / S}$ and $\tilde{Y}$ the universal family on $X \times{ }_{S} D$. $\mathcal{O}_{X}(1)$ defines a section $q: S \rightarrow P$ and $\left(D \times{ }_{P} S, \tilde{Y} \times_{P} S\right)$ parametrizes all the members of $\left|\mathcal{O}_{X_{s}}(1)\right|, s \in S$. Let $S^{\prime}$ be the maximal open set of $D \times{ }_{P} S$ over which $\tilde{Y} \times{ }_{P} S$ is smooth and 
set $X^{\prime}=\left.\left(\tilde{Y} \times_{P} S\right)\right|_{s^{\prime}}$. Thus we obtain a smooth, projective, geometrically integral morphism $f^{\prime}: X^{\prime} \rightarrow S^{\prime}$ of $\Lambda$-schemes which parametrizes all the smooth members of $\left|\mathcal{O}_{x_{s}}(1)\right|, s \in S$

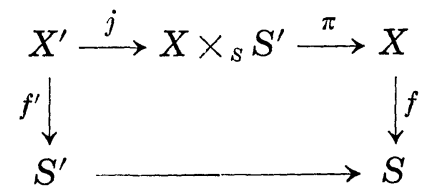

and $\mathcal{O}_{X^{\prime}}(1)=j^{*} \pi^{*}\left(\mathcal{O}_{X}(1)\right)$ is very ample over $S^{\prime}$.

Proposition 3.6. Let $\mathscr{F}\left(\right.$ or, $\left.\mathscr{F}^{\prime}\right)$ be a subfamily of $\Sigma_{X / S}\left(n, r, a_{1}, \cdots, a_{n}\right)$ (or, $\Sigma_{X / S}^{\prime}\left(n, r, a_{1}, a_{2}\right)$, resp.). Assume that if $E$ is a coherent sheaf on $X_{s}$ contained in $\mathscr{F}\left(\right.$ or, $\left.\mathscr{F}^{\prime}\right)$, then for general members $Y$ of $\left|\mathcal{O}_{X}(1)\right|,\left.E\right|_{Y}$ is $\mu$ semi-stable. If $B_{n-1, r}(\Lambda)$ (or, $B_{n-1, r}^{\prime}(\Lambda)$, resp.) is true, then $\mathscr{F}$ (or, $\mathscr{F}$, resp.) is bounded.

Proof. Let $E \in \mathscr{F}$ (or, $\left.\mathscr{F}^{\prime}\right)$ and $\sigma$ a general element of $H^{\circ}\left(X_{s}, \mathcal{O}_{X_{s}}(1)\right)$. We have the exact sequence

$$
\left.0 \longrightarrow E(-1) \longrightarrow E \longrightarrow E\right|_{Y} \longrightarrow 0
$$

where $Y$ is the zero scheme of $\sigma$.

Lemma 3.7. (1) If $E$ satisfies $\left(S_{2}\right)$, then $H^{0}\left(X_{s}, E(-\ell)\right)=0=H^{1}\left(X_{s}, E(-\ell)\right)$ for sufficiently large $\ell$.

(2) If $\sigma$ is sufficiently general and if $E$ satisfies $\left(S_{2}\right)$, then $\left.E\right|_{Y}$ satisfies $\left(S_{2}\right)$, too.

Proof. There exists a resolution of $E$ by locally free, coherent sheaves

$$
0 \longrightarrow E_{n-2} \stackrel{f_{n-2}}{\longrightarrow} E_{n-3} \stackrel{f_{n-3}}{\longrightarrow} \cdots \stackrel{f_{1}}{\longrightarrow} E_{0} \stackrel{f_{0}}{\longrightarrow} E \longrightarrow 0
$$

and $E$ satisfies $\left(S_{2}\right)$ if and only if for the set of pinch points $Y_{i}$ of $\operatorname{ker}\left(f_{i}\right)$, $\operatorname{dim} Y_{i} \leq n-i-4$ (cf. [8] Proof of Lemma 2.2).

(1) This is well-known (see [11]). We shall give a proof for completeness. Since every $E_{i}$ is locally free and coherent, there is an integer $\ell_{0}$ such that for all $\ell \geq \ell_{0}, H^{j}\left(X_{s}, E_{i}(-\ell)\right)=0,0 \leq j<n, 0 \leq i \leq n-2$. Set $\operatorname{ker}\left(f_{i}\right)=K_{i}$. Then by induction on $i$ and the exact sequences

$$
0 \longrightarrow K_{i} \longrightarrow E_{i} \longrightarrow K_{i-1} \longrightarrow 0 \text {, }
$$

we have $H^{j}\left(X_{s}, K_{i}(-\ell)\right)=0$ for $0 \leq j \leq i+2$ and $\ell \geq \ell_{0}$. Thus, from the exact sequence 


$$
0 \longrightarrow K_{0} \longrightarrow E_{0} \longrightarrow E \longrightarrow 0
$$

we have $H^{0}\left(X_{s}, E(-\ell)\right)=0=H^{1}\left(X_{s}, E(-\ell)\right)$ for all $\ell \geq \ell_{0}$.

(2) If $\sigma$ is sufficiently general, then

$$
\left.\left.\left.\left.0 \longrightarrow E_{n-2}\right|_{Y} \longrightarrow E_{n-3}\right|_{Y} \longrightarrow \cdots \longrightarrow E_{0}\right|_{Y} \longrightarrow E\right|_{Y} \longrightarrow 0
$$

is exact and $\operatorname{dim}\left(Y_{i} \cap Y\right) \leq n-i-5$. Since the set of pinch points of $\operatorname{ker}\left(\left.f_{i}\right|_{Y}\right)$ is $Y \cap Y_{i}$, we see that for the above $Y,\left.E\right|_{Y}$ satisfies $\left(S_{2}\right)$. q.e.d.

Now let us come back to the proof of Proposition 3.6. The exact sequence (3.6.1) implies that $\chi\left(\left(\left.E\right|_{Y}\right)(m)\right)=\Delta \chi(E(m))=\chi(E(m))-\chi(E(m-1))$. This and the above lemma show that if $\left.E\right|_{Y}$ is $\mu$-semi-stable, then $\left.E\right|_{Y} \in$ $\Sigma_{X^{\prime} / S^{\prime}}\left(n-1, r, a_{1}, \cdots, a_{n-1}\right)$ or $\Sigma_{X^{\prime} / S^{\prime}}^{\prime}\left(n-1, r, a_{1}, a_{2}\right)$ according as $E \in \mathscr{F}$ or $E \in \mathscr{F}^{\prime}$. Replacing $\mathscr{F}$ (or, $\mathscr{F}^{\prime}$ ) by $\mathscr{F}(m)=\{E(m) \mid E \in \mathscr{F}\} \quad$ (or, $\mathscr{F}^{\prime}(m)=$ $\left\{E(m) \mid E \in \mathscr{F}^{\prime}\right\}$, resp.), $m \gg 0$, we may assume that (i) $H^{i}\left(Y,\left(\left.E\right|_{Y}\right)(\ell)\right)=0$ for all $i>0$, all $\ell \geq 0$ and all $E \in \mathscr{F}$ (or, $E \in \mathscr{F}^{\prime}$, resp.), (ii) $\operatorname{dim} H^{\circ}\left(Y,\left(\left.E\right|_{Y}\right)(\ell)\right.$ ) $\leq c_{\ell}, \operatorname{dim} H^{1}\left(Y,\left(\left.E\right|_{Y}\right)(\ell)\right) \leq c_{\ell}^{\prime}$ for all $E \in \mathscr{F}$ (or, $E \in \mathscr{F}^{\prime}$, resp.) and all $\ell$, (iii) $\sharp\left\{\Delta \chi(E(m)) \mid E \in \mathscr{F}\right.$ (or, $E \in \mathscr{F}^{\prime}$, resp.) $\}<\infty$ and (iv) there is a sequence (b) $=\left(b_{1}, \cdots, b_{n}\right)$ of integers such that $\left.E\right|_{Y}$ is $(b)$-sheaf for all $E \in \mathscr{F}$ (or, $E \in \mathscr{F}^{\prime}$, resp.) (see [6] Definition 1.5) because $B_{n-1, r}(\Lambda)$ (or, $B_{n-1, r}^{\prime}(\Lambda)$, resp.) is true. Moreover, in the case of $\mathscr{F}^{\prime}$, we may assume that (v) there is an integer $\ell_{0}$ such that $H^{0}\left(Y,\left(\left.E\right|_{Y}\right)(-\ell)\right)=0=H^{1}\left(Y,\left(\left.E\right|_{Y}\right)(-\ell)\right)$ for all $\ell \geq \ell_{0}$ and all $E \in \mathscr{F}^{\prime}$. The exact sequence (3.6.1) and (i) yield that $H^{i}\left(X_{s}, E\right) \cong H^{i}\left(X_{s}, E(\ell)\right)$ for all $i \geq 2$ and $\ell \geq 0$, whence $H^{i}\left(X_{s}, E\right)=0$ for all $i \geq 2$. Therefore,

(3.6.2) $\operatorname{dim} H^{0}\left(X_{s}, E\right)-\operatorname{dim} H^{1}\left(X_{s}, E\right)=\chi(E)=r d+a_{1}+d_{2}+\cdots+d_{n}$, where $\chi(E(m))=r d\left(\begin{array}{c}m+n \\ n\end{array}\right)+a_{1}\left(\begin{array}{c}m+n-1 \\ n-1\end{array}\right)+\sum_{i=2}^{n} d_{i}\left(\begin{array}{c}m+n-\mathrm{i} \\ n-i\end{array}\right)$.

On the other hand, $\operatorname{dim} H^{0}\left(X_{s}, E(\ell)\right)-\operatorname{dim} H^{0}\left(X_{s}, E(\ell-1)\right) \leq \operatorname{dim} H^{0}\left(Y,\left(\left.E\right|_{Y}\right)(\ell)\right)$ $\leq c_{\ell}$ and since $E$ is $\mu$-semi-stable, $H^{0}\left(X_{s}, E(\ell)\right)=0$ if $d\left(E(\ell), \mathcal{O}_{X_{s}}(1)\right)<0$. Thus, $\operatorname{dim} H^{0}\left(X_{s}, E\right) \leq c_{0}+c_{-1}+\cdots+c_{-\alpha}=b_{0}$, where $\alpha$ is independent of the choice of $E$. Combining this with (3.6.2), we have

$$
b_{0} \geq \operatorname{dim} H^{0}\left(X_{s}, E\right) \geq r d+a_{1}+d_{2}+\cdots+d_{n} .
$$

By virtue of (iii) above, $d_{2}, \cdots, d_{n-1}$ range over a finite set and hence, $d_{n}$ is bounded from above. In the case of $\mathscr{F}, d_{n}$ is bounded because $d_{n} \geq a_{n}$. Thus $\sharp\{\chi(E(m)) \mid E \in \mathscr{F}\}<\infty$. Assume that $E$ is contained in $\mathscr{F}^{\prime}$. The exact sequence (3.6.1) shows that $\operatorname{dim} H^{1}\left(X_{s}, E(\ell)\right)-\operatorname{dim} H^{1}\left(X_{s}, E(\ell-1)\right)$ 
$\leq \operatorname{dim} H^{1}\left(Y,\left(\left.E\right|_{Y}\right)(\ell)\right) \leq c_{\ell}^{\prime}$. (v) implies that, for $\ell \geq \ell_{0}$ and all $E \in \mathscr{F}^{\prime}$, $H^{1}\left(X_{s}, E(-\ell)\right) \cong H^{1}\left(X_{s}, E\left(-\ell_{0}\right)\right)$. By virtue of this and Lemma 3.7, we have that $H^{1}\left(X_{s}, E(-\ell)\right)=0$ for all $\ell \geq \ell_{0}$ and all $E \in \mathscr{F}^{\prime}$. Therefore,

$$
0 \leq \operatorname{dim} H^{1}\left(X_{s}, E\right) \leq c_{0}^{\prime}+c_{-1}^{\prime}+\cdots+c_{1-\ell_{0}}^{\prime}=c .
$$

This and (3.6.2) imply that

$$
-c \leq r d+a_{1}+d_{2}+\cdots+d_{n} .
$$

Since $d_{2}, \cdots, d_{n-1}$ range over a finite set, $d_{n}$ is bounded from below. Thus, $d_{n}$ is bounded in the case of $\mathscr{F}^{\prime}$, too. Hence $\sharp\left\{\chi(E(m)) \mid E \in \mathscr{F}^{\prime}\right\}$ $<\infty$. On the other hand, (iv) and (3.6.3) show that $E \in \mathscr{F}$ (or, $E \in \mathscr{F}^{\prime}$ ) is $\left(b_{0}, b_{1}, \cdots, b_{n}\right)$-sheaf. Then, the boundedness of $\mathscr{F}$ or $\mathscr{F}^{\prime}$ follows from Théorème 1.13 of [6].

q.e.d.

Using the above proposition, we have

Proposition 3.8. $B_{n, 1}(\Lambda)$ and $B_{n, 1}^{\prime}(\Lambda)$ hold for all $n$ and $\Lambda$ and hence, $B_{n, 1}^{\prime \prime}(\Lambda)$ is true for all $n$ and $\Lambda$.

Proof. $\Sigma_{X / S}\left(1,1, a_{1}\right)=\Sigma_{X / s}^{\prime}\left(1,1, a_{1}, a_{2}\right)$ is the family of the classes of invertible sheaves on curves with fixed degree. Then, its boundedness is well-known. Pick an $L$ in $\Sigma_{X / S}\left(n, 1, a_{1}, \cdots, a_{n}\right)$ or $\Sigma_{X / S}^{\prime}\left(n, 1, a_{1}, a_{2}\right)$. For general $Y$ of $\left|\mathcal{O}_{X_{s}}(1)\right|,\left.L\right|_{Y}$ is torsion free or equivalently $\mu$-semi-stable. Using induction on $n$, our assertion follows from Proposition 3.6. q.e.d.

Remark 3.9. Proposition 3.8 is a special case of Théorème 3.13 of [6].

We have proved the boundedness of the family of semi-stable sheaves with fixed Chern classes on a surface ([7] Theorem 2.5 and Corollary 2.5.3). Using Proposition 3.8 instead of Step B in the proof of Theorem 2.5 of [7], the same method is applicable to proving that a similar assertion to Theorem 2.5 of [7] holds for the families $\Sigma_{X / S}\left(2, r, a_{1}, a_{2}\right)$. Then, as the proofs Corollary 2.5.2 and Corollary 2.5.3 of [7], we can show that $B_{2, r}(\Lambda)$ holds. In this case, $B_{2, r}(\Lambda)$ is stronger than $B_{2, r}^{\prime}(\Lambda)$.

ThEOREM 3.10. $B_{2, r}(\Lambda)$ and $B_{2, r}^{\prime}(\Lambda)$ hold and hence, $B_{2, r}^{\prime \prime}(\Lambda)$ does for all $r$ and $\Lambda$.

This and Theorem 3.1 yield

Theorem 3.11. $B_{n, 2}(\Lambda)$ and $B_{n, 2}^{\prime}(\Lambda)$ hold and hence, $B_{n, 2}^{\prime \prime}(\Lambda)$ is true for all $n$ and $\Lambda$. 
Proof. The case of $n=1$ is well-known and the case of $n=2$ is a special case of Theorem 3.10. Assume $n \geq 3$ and take a $\mu$-semi-stable sheaf $E$ of rank 2 on a fibre $X_{s}$. By virtue of Theorem 3.1, for general member $Y$ of $\left|\mathcal{O}_{X_{s}}(1)\right|,\left.E\right|_{Y}$ is $\mu$-semi-stable. Thus, applying Proposition 3.6 to $\mathscr{F}=\Sigma_{X / S}\left(n, 2, a_{1}, \cdots, a_{n}\right)$ and $\mathscr{F}^{\prime}=\Sigma_{X / S}^{\prime}\left(n, 2, a_{1}, a_{2}\right)$, we see that $\Sigma_{X / S}\left(n, 2, a_{1}, \cdots, a_{n}\right)$ and $\Sigma_{X / S}^{\prime}\left(n, 2, a_{1}, a_{2}\right)$ are bounded. q.e.d.

\section{§4. A technique of Barth*)}

Let $X$ be a non-singular projective surface over an algebraically closed field $k, \mathcal{O}_{X}(1)$ a very ample invertible sheaf on $X$ and $L$ a very ample linear subsystem of the complete linear system $\left|\mathcal{O}_{X}(1)\right|$. As in the first paragraph of $\S 2$, we have the following diagram:

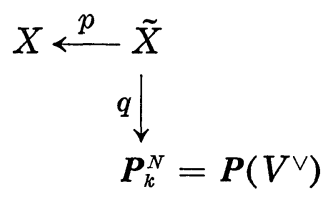

where $V$ is the linear subspace of $H^{\circ}\left(X, \mathcal{O}_{X}(1)\right)$ defining $L$ and $q: \tilde{X} \rightarrow \boldsymbol{P}_{k}^{N}$ parametrizes all the divisors of $L$. Note that $p$ is a $\boldsymbol{P}^{N-1}$-bundle and hence, $\tilde{X}$ is smooth.

Let $E$ be a locally free, coherent sheaf on $X$ of rank $r$. Assume the following:

(4.1.1) $E$ is $\mu$-semi-stable with respect to $\mathcal{O}_{X}(1)$.

(4.1.2) For the generic point $u$ of $\boldsymbol{P}_{k}^{N}, p^{*}(E) \otimes k(u)=\bar{E}$ contains subbundle $\bar{F}$ of rank $r-1$ such that $\mu(\bar{F})>\mu(\bar{E})$.

As in the proof of Lemma 2.2, there is a non-empty open set $U$ of $\boldsymbol{P}_{k}^{N}$ and a coherent subsheaf $\tilde{F}_{0}$ of $p^{*}(E)_{U}$ such that $\tilde{F}_{0} \otimes k(u)=\bar{F}$ and $p^{*}(E)_{U} / \tilde{F}_{0}$ is $U$-flat. Then, on a non-empty open set $W$ of $U,\left(\tilde{F}_{0}\right)_{W}$ is a subbundle of $p^{*}(E)_{W}$. Furthermore, there is a coherent subsheaf $\tilde{F}$ of $p^{*}(E)=\tilde{E}$ such that $\tilde{F}_{U}=\tilde{F}_{0}$ and $\tilde{E} / \tilde{F}$ is torsion free.

$\left.\boldsymbol{P}\left(\tilde{F}^{\vee}\right)\right|_{X_{W}}$ is a projective subbundle of $\left.\boldsymbol{P}\left(\tilde{E}^{\vee}\right)\right|_{X_{W}}$. Let $\Gamma$ be the closure of $\left.\boldsymbol{P}\left(\tilde{F}^{\vee}\right)\right|_{X_{W}}$ in $\boldsymbol{P}\left(\tilde{E}^{\vee}\right)$. If $\Gamma$ is regarded as a subscheme of $\boldsymbol{P}\left(\tilde{E}^{\vee}\right)$ with reduced structure, then it is a divisor of $\boldsymbol{P}\left(\tilde{E}^{\vee}\right)$. Since $\boldsymbol{P}\left(\tilde{E}^{\vee}\right)=\boldsymbol{P}\left(E^{\vee}\right) \times_{x} \tilde{X}$, we have the following commutative diagram;

*) The argument of this section is a modification of some parts of $\S 4$ and $\S 5$ of [2]. 


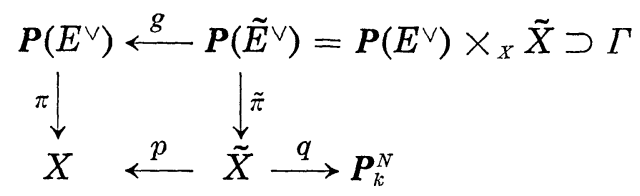

LEMMA 4.2. $g(\Gamma)=\boldsymbol{P}\left(E^{\vee}\right)$.

Proof. Assume that $g(\Gamma) \neq \boldsymbol{P}\left(E^{\vee}\right)$, then $g(\Gamma)$ is an irreducible divisor of $\boldsymbol{P}\left(E^{\vee}\right)$ because $\Gamma$ is a projective subbundle of $\boldsymbol{P}\left(\tilde{E}^{\vee}\right)$ at general points of $\tilde{X}$ and a divisor of $\boldsymbol{P}\left(\tilde{E}^{\vee}\right)$. Moreover, for a general point $x$ of $X, g(\Gamma)_{x}$ is a hyperplane of $\boldsymbol{P}\left(E^{\vee}\right)_{x}=\boldsymbol{P}^{r-1}$. Thus, $g(\Gamma)$ is a projective subbundle of $\boldsymbol{P}\left(E^{\vee}\right)$ outside a finite set of points $\left\{x_{1}, \cdots, x_{t}\right\}$ of $X$. This means that there is a quotient bundle $G$ of $\left.E^{\vee}\right|_{X_{0}}$ such that $P(G)=g(\Gamma)_{X_{0}}$, where $X_{0}=X-\left\{x_{1}, \cdots, x_{t}\right\} . \quad$ Therefore, $\quad \boldsymbol{P}(G) \times_{X} \tilde{X}=\Gamma_{p-1\left(X_{0}\right)}$ and hence, $\left(\left.p\right|_{X_{0}}\right)^{*}\left(G^{\vee}\right)_{W}=\tilde{F}_{W}$. Let $C$ be a smooth curve which passes through none of $x_{1}, \cdots, x_{t}$ and corresponds to a point $v$ of $W \subseteq \boldsymbol{P}_{k}^{N}$. Then, $C \subset p^{-1}\left(X_{0}\right)$ and $\mu(F \otimes k(v))=\mu(\bar{F})>\mu(\bar{E})=\mu(E)$. Let $G^{\prime}$ be a coherent subsheaf of $E$ such that $\left.G^{\prime}\right|_{x_{0}}=G^{\vee}$. For this $G^{\prime}$, we have

$$
\mu\left(G^{\prime}\right)=\mu\left(\left.G^{\prime}\right|_{c}\right)=\mu\left(\left.G^{\vee}\right|_{c}\right)=\mu(F \otimes k(v))=\mu(\bar{F})>\mu(E),
$$

which contradicts the assumption (4.1.1).

q.e.d.

Now let us assume

(4.3) $k$ is a field of characteristic zero.

Then, since $\Gamma$ is a variety and $g$ is surjective, $\left.g\right|_{r}$ is smooth on a nonempty open set $\Delta$ of $\Gamma_{\mathrm{W}}$. Pick a $k$-rational point $y$ of the open set $q \tilde{\pi}(\Delta)$ such that $C=\tilde{X}_{v}$ is smooth. $C$ is regarded as a curve in $X$, too. $\Gamma$ cuts out a projective subbundle $A=\boldsymbol{P}\left(\tilde{F}^{\vee} \otimes k(y)\right)$ on $\boldsymbol{P}\left(\tilde{E}^{\vee}\right)_{c} . \quad B=g(A)$ is also a projective subbundle of $\boldsymbol{P}\left(E^{\vee}\right)_{c}$ and $g$ induces isomorphisms of $\boldsymbol{P}\left(\tilde{E}^{\vee}\right)_{C}$ to $\boldsymbol{P}\left(E^{\vee}\right)_{C}$ and of $A$ to $B$.

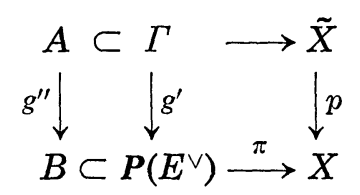

From these we obtain the following commutative diagram of normal bundles; 


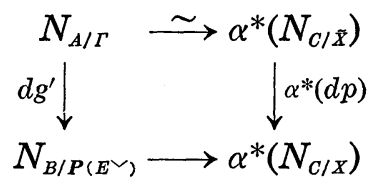

where $\alpha$ is the projection of $A$ to $C$ which is identified with the projection of $B$ to $C$ (note that $\Gamma$ is smooth along $A$ ). Since $g^{\prime}$ is smooth on $\Delta$ and $C \cap \Delta \neq \phi, d g^{\prime}$ is generically surjective. It is easy to see that $N_{C / \tilde{X}}=V^{\prime} \otimes_{k} \mathcal{O}_{c}$ and $N_{C / X}=\mathcal{O}_{C}(1)=\left.\mathcal{O}_{X}(1)\right|_{c}$, where $V^{\prime}=\operatorname{im}(\theta)$ defined by the homomorphism

$$
\theta: V \longrightarrow H^{\circ}\left(X, \mathcal{O}_{X}(1)\right) \longrightarrow H^{\circ}\left(C, \mathcal{O}_{C}(1)\right) .
$$

Moreover, the map $d p$ is identified with the natural homomorphism

$$
V^{\prime} \otimes_{k} \mathcal{O}_{C} \longrightarrow H^{\circ}\left(C, \mathcal{O}_{C}(1)\right) \otimes_{k} \mathcal{O}_{C} \longrightarrow \mathcal{O}_{C}(1) \text {. }
$$

Thus, we obtain the following exact commutative diagram

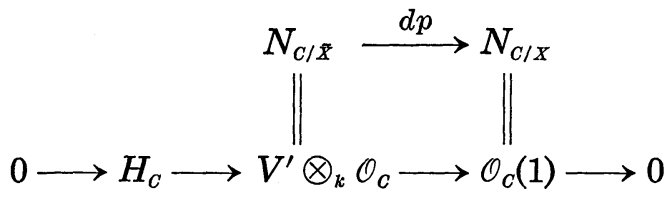

where $H_{C}$ is a locally free sheaf of rank $N-1$ which is uniquely determined by $C$. On the other hand, the following exact commutative diagram is obtained;

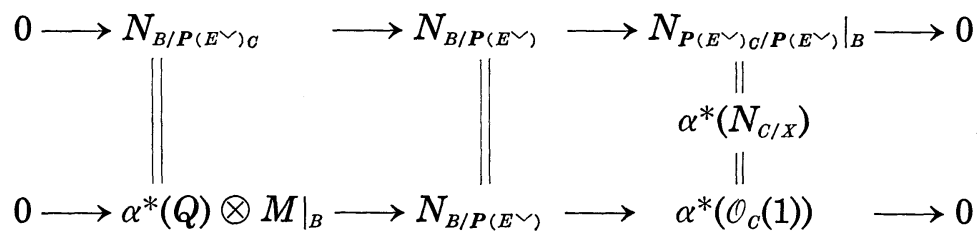

where $Q=\left.E\right|_{c} / \tilde{F} \otimes k(y)$ and $M$ is the tautological line bundle of $\left.E^{\vee}\right|_{c}$ on $\boldsymbol{P}\left(E^{\vee}\right)_{c}$. The above three diagrams provide us with following exact commutative diagram;

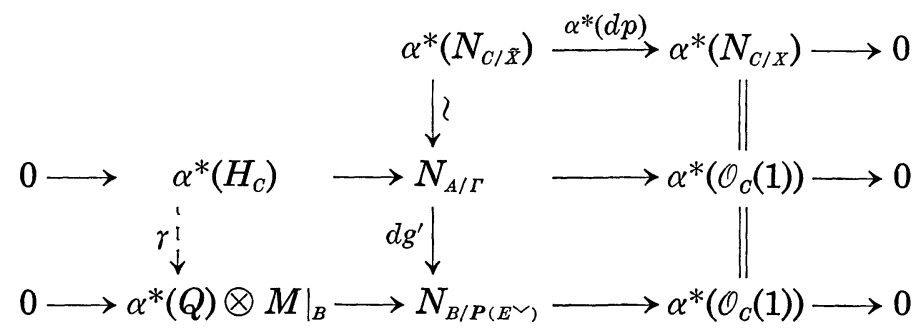


Since $d g^{\prime}$ is generically surjective, so is $\gamma$. We have, therefore,

$$
0 \neq \gamma \in \operatorname{Hom}_{o_{B}}\left(\alpha^{*}\left(H_{C}\right),\left.\alpha^{*}(Q) \otimes M\right|_{B}\right)=\operatorname{Hom}_{\odot_{C}}\left(H_{C}, Q \otimes(\tilde{F} \otimes k(y))^{\vee}\right) .
$$

LEMMA 4.5. Every non-trivial coherent subsheaf $G$ of $H_{C}$ has a negative degree.

Proof. If $N=2$, then $H_{C} \cong \mathcal{O}_{c}(-1)$. Our assertion is obvious in this case. Assume that $N>2$. We may assume that $G$ is a subbundle of $H_{C}$. Since $H_{C}$ is a subbundle of $\mathcal{O}_{C}^{\oplus N}$, so is $G$. Therefore, $\operatorname{deg} G=\operatorname{deg}(\operatorname{det} G)$ $\leq 0$. If $\operatorname{deg} G=0$, then $G^{\vee}$ is a quotient sheaf of $\mathcal{O}_{C}^{\oplus N}$ whose degree is zero. Thus $G^{\vee} \cong \mathcal{O}_{C}^{\oplus s}$, that is, $G \cong \mathcal{O}_{C}^{\oplus s}$. This implies that $\operatorname{dim} H^{\circ}\left(C, H_{C}\right)$ $\geq \operatorname{dim} H^{\circ}(C, G)=s>0$. On the other hand, we have the exact sequence

$$
0 \longrightarrow H^{0}\left(C, H_{C}\right) \longrightarrow H^{\circ}\left(C, \mathcal{O}_{C}^{\oplus N}\right) \stackrel{\delta}{\longrightarrow} H^{\circ}\left(C, \mathcal{O}_{C}(1)\right)
$$

and by the definition of $H_{C}, \delta$ is injective. Hence $H^{\circ}\left(C, H_{C}\right)=0$. This is a contradiction. Therefore, $\operatorname{deg} G<0$.

q.e.d.

Let $E$ be a coherent torsion free sheaf on a non-singular projective surface $X$ with very ample invertible sheaf $\mathcal{O}_{X}(1) . \quad E$ is a subsheaf of a locally free sheaf $E^{\prime}$ with $\operatorname{dim}\left(\operatorname{Supp}\left(E^{\prime} \mid E\right)\right) \leq 0$. Such an $E^{\prime}$ is unique up to isomorphisms. Assume that $r(E)=2$. For a non-singular curve $C$ in $L$, set

$d(E, C)=\min \left\{\operatorname{deg}\left(\left(\left(\left.E^{\prime}\right|_{c}\right) / D\right) \otimes D^{\vee}\right)=\operatorname{deg} E-2 \operatorname{deg} D \mid D:\right.$ line subbundle of $\left.\left.E^{\prime}\right|_{c}\right\}$ and

$$
d(E)=\max \{d(E, C) \mid C: \text { non-singular curve in } L\} .
$$

Then, there exists a non-empty open set $U$ of $L$ such that $d(E)=d(E, C)$ for all $C$ in $U$.

THEOREM 4.6. Let $X$ be a non-singular projective surface over an algebraically closed field $k$ of characteristic zero, $\mathcal{O}_{X}(1)$ a very ample invertible sheaf on $X$ and $L$ a very ample linear subsystem of $\left|\mathcal{O}_{X}(1)\right|$.

(1) Let $E$ be a coherent sheaf of rank 2 on $X$. Assume that $E$ is $\mu$ semi-stable with respect to $\mathcal{O}_{X}(1)$.

(a) If $X=\boldsymbol{P}^{2}$ and $\mathcal{O}_{X}(1)$ is the line bundle corresponding to lines of $\boldsymbol{P}^{2}$, then $d(E) \geq-1$.

(b) Otherwise, $d(E)>-C^{2}$ for a $C \in L$.

(2) Let $E$ be a coherent sheaf on $X$ having the properties (4.1.1) and 
(4.1.2). Then, $(\bar{E} / \bar{F}) \otimes \bar{F}^{\vee}$ contains a non-zero subbundle $M$ with $\operatorname{deg} M$ $>-C^{2}$ for a $C \in L$ and $r(M)<N-1$ or with $\operatorname{deg} M \geq-C^{2}$ and $r(M)=$ $N-1$.

Proof. Replacing $E$ by $E^{\prime}$ defined just before this theorem, we may assume that $E$ is locally free.

(1) If, for every general member $C$ of $L,\left.E\right|_{C}$ is $\mu$-semi-stable, then our assertion is trivial. Otherwise, for the generic member $D$ of $L$, we have the Harder-Narasimhan filtration $\left.0 \subsetneq \bar{F} \subsetneq E\right|_{D}$ of $\left.E\right|_{D}$. For this $\bar{F}$, we have the property (4.1.2). If $C$ is general, $\left.E\right|_{C}$ has the HarderNarasimhan filtration $\left.0 \subseteq F \sqsubseteq E\right|_{c}$. Moreover, when $C$ is sufficiently general, $\operatorname{Hom}_{\sigma_{C}}\left(H_{C}, \operatorname{det}\left(\left.E\right|_{C}\right) \otimes F^{\otimes(-2)}\right) \neq 0$ by (4.4). If $N=2$, then $H_{C}=$ $\mathcal{O}_{C}(-1)$, whence $\operatorname{deg} E-2 \operatorname{deg} F \geq-C^{2}$. This case can happen only when $X=\boldsymbol{P}^{2}$ and $\mathcal{O}_{X}(1)$ is the invertible sheaf corresponding to lines of $\boldsymbol{P}^{2}$. Assume that $N>2$. By Lemma 4.5, every quotient coherent sheaf of $H_{C}$ has a degree $>-C^{2}$. Therefore, $\operatorname{deg} E-2 \operatorname{deg} F>-C^{2}$. If $C$ is sufficiently general, $d(E)=\operatorname{deg} E-2 \operatorname{deg} F$ because $F$ is a filter of the Harder-Narasimhan filtration of $\left.E\right|_{c}$. Thus our proof is completed.

(2) A similar argument to the above is applicable to this case. Hence, we omit the proof.

Remark 4.7. Theorem 4.6 is false unless we assume that the characteristic of $k$ is zero. For example, on $\boldsymbol{P}^{2}, T_{\boldsymbol{P}^{2}}^{\left(p^{r}\right)}$ is $\mu$-semi-stable and $\left.T_{P^{2}}^{\left(p^{r}\right)}\right|_{e}$ $=\mathcal{O}_{\ell}\left(p^{r}\right) \oplus \mathcal{O}_{\ell}\left(2 p^{r}\right)$ for every line $\ell$ of $\boldsymbol{P}^{2}$, where $T_{P^{2}}^{\left(p^{r}\right)}$ is the pull-back of the tangent bundle $T_{P^{2}}$ of $\boldsymbol{P}^{2}$ by $r$-th Frobenius morphism.

ExAmple 4.8. (1) Let us consider the case where $X=\boldsymbol{P}^{2}, \mathcal{O}_{X}(1)=$ $\mathcal{O}_{P^{2}}(2)$. For general member $C$ of $\left|\mathcal{O}_{X}(1)\right|$, we have the exact sequence defining $H_{C}$

$$
0 \longrightarrow H_{C} \longrightarrow V^{\prime} \otimes_{k} \mathcal{O}_{C} \stackrel{\zeta}{\longrightarrow} \mathcal{O}_{c}(1) \longrightarrow 0 \text {. }
$$

In this case, $H^{0}(\zeta)$ is bijective and $H^{1}\left(C, V^{\prime} \otimes_{k} \mathcal{O}_{C}\right)=0$. Thus $H^{0}\left(C, H_{C}\right)$ $=0=H^{1}\left(C, H_{C}\right)$. For a quotient line bundle $L$ of $H_{C}, H^{1}(C, L)=0$ because $H^{1}\left(C, H_{C}\right)=0$. Thus $\operatorname{deg} L \geq-1$. Therefore, $d(E) \geq-1$ for every $\mu$ semi-stable sheaf $E$ of rank 2 on $\boldsymbol{P}^{2}$ (see the proof of Theorem 4.6). On the other hand, $d\left(E, \mathcal{O}_{X}(1)\right)$ is even and every smooth member of $\left|\mathcal{O}_{X}(1)\right|$ is isomorphic to $\boldsymbol{P}^{1}$. Thus we see that $d(E)=0$ (cf. [2] p. 137).

(2) Let us consider the case of $\operatorname{rank} 3$ on $\left(\boldsymbol{P}^{2}, \mathcal{O}_{P^{2}}(1)\right)$. Take a $\mu$ - 
semi-stable sheaf $E$ of rank 3 on $\boldsymbol{P}^{2}$ and a general line $C=\boldsymbol{P}^{1} .\left.\quad E\right|_{c}$ is isomorphic to $\mathcal{O}_{P_{1}}\left(a_{1}\right) \oplus \mathcal{O}_{P_{1}}\left(a_{2}\right) \oplus \mathcal{O}_{P_{1}}\left(a_{3}\right)$ with $a_{1} \geq a_{2} \geq a_{3}$.

(i) If $a_{2}>a_{3}$, then we see that $\mathcal{O}_{P^{1}}\left(a_{3}-a_{1}\right) \oplus \mathcal{O}_{P 1}\left(a_{3}-a_{2}\right)$ contains a line subbundle of degree $\geq-1$ by Theorem 4.6, (2). Thus $a_{3}-a_{2} \geq-1$.

(ii) If $a_{1}>a_{2}$, then we see $a_{2}-a_{1}>-1$ by applying Theorem 4.6, (2) to the dual sheaf $E^{\vee}$ of $E$.

After twisting suitably, we may assume that $d=d\left(E, \mathcal{O}_{\boldsymbol{P}^{2}}(1)\right)=0,1$ or 2. By (i) and (ii) above, we have

(a) if $d=0$, then $\left(a_{1}, a_{2}, a_{3}\right)=(1,0,-1)$ or $(0,0,0)$,

(b) if $d=1$, then $\left(a_{1}, a_{2}, a_{3}\right)=(1,0,0)$,

(c) if $d=2$, then $\left(\left(a_{1}, a_{2}, a_{3}\right)=(1,1,0)\right.$.

Can all the cases happen?

\section{$\S 5$. Boundedness of semi-stable sheaves of rank 3 and 4}

We shall begin with the following.

Lemma 5.1. Assume that $\sum_{X / S}^{\prime}\left(n, r, a_{1}, a_{2}\right)$ is bounded (for the notation, see $\S 3)$. Let $\mathscr{F}$ be the family of the classes of coherent sheaves on the fibres of $X$ over $S$ such that $E$ is a member of $\mathscr{F}$ if and only if $r(E)=r$, $a_{1}(E)=a_{1}$ and $E$ is $\mu$-semi-stable. Then, $\left\{a_{2}(E) \mid E \in \mathscr{F}\right\}$ is bounded from above.

Proof. Assume that a coherent sheaf $E$ on a fibre $X_{s}$ is contained in $\mathscr{F}$. Let $Y$ be the set of pinch points of $E$ and let $i$ the open immersion of $X-Y$ to $X$. Then, $E^{\prime}=i_{*} i^{*}(E)$ satisfies $\left(S_{2}\right), a_{1}(E)=a_{1}\left(E^{\prime}\right), E^{\prime}$ is $\mu$-semi-stable and $a_{2}\left(E^{\prime}\right) \geq a_{2}(E)$ (see the proof of Lemma 3.5). Thus we may replace $\mathscr{F}$ by $\mathscr{F}^{\prime}=\left\{E \in \mathscr{F} \mid E\right.$ satisfies $\left.\left(S_{2}\right)\right\}$. If $E \in \mathscr{F}^{\prime}$ is not contained in $\Sigma_{X / S}^{\prime}\left(n, r, a_{1}, a_{2}\right)$, then $a_{2}>a_{2}(E)$. Since $\Sigma_{X / S}^{\prime}\left(n, r, a_{1}, a_{2}\right)$ is bounded, $\#\left\{\chi(E(m)) \mid E \in \Sigma_{X / S}^{\prime}\left(n, r, a_{1}, a_{2}\right)\right\}<\infty$, a fortiori, $\left\{a_{2}(E) \mid E \in \Sigma_{X / S}^{\prime}\left(n, r, a_{1}, a_{2}\right)\right\}$ is bounded from above by an integer $b$. Therefore, $\left\{a_{2}(E) \mid E \in \mathscr{F}^{\prime}\right\}$ is bounded from above by $b$.

q.e.d.

Let $X$ be a smooth, projective, integral scheme over an algebraically closed field $k$ of characteristic zero. $L$ a very ample linear subsystem of a complete linear system $\left|\mathcal{O}_{X}(1)\right|$ and $E$ a torsion free coherent sheaf on $X$. $\left.E\right|_{D}$ is not $\mu$-semi-stable with respect to $\left.\mathcal{O}_{X}(1)\right|_{D}$ for the generic member $D$ of $L$ if and only if $\left.E\right|_{Z}$ is not $\mu$-semi-stable with respect to $\mathcal{O}_{Z}(1)$ $=\left.\mathcal{O}_{X}(1)\right|_{Z}$ for every $k$-rational member $Z$ of a non-empty open subset of 
$L$. Thus we may say that $\left.E\right|_{Y}$ is not $\mu$-semi-stable for every general member $Y$ of $L$.

Lemma 5.2. Under the above situation, assume that $r(E)=\operatorname{dim} X \geq 2$, $E$ is $\mu$-semi-stable and $\left.E\right|_{Z}$ is not $\mu$-semi-stable with respect to $\mathcal{O}_{Z}(1)$ for every general member $Z$ of $L$. Then, for general members $Z$ of $L,\left.E\right|_{Z}$ has the Harder-Narasimhan filtration

$$
0=E_{0} \sqsubseteq E_{1} \subsetneq E_{2}=\left.E\right|_{z}
$$

such that (1) $r\left(E_{1}\right)=1$ or $r(E)-1$ and (2) $-d \leq r\left(E_{1}\right) d\left(E, \mathcal{O}_{X}(1)\right)-$ $r(E) d\left(E_{1}, \mathcal{O}_{X}(1)\right)<0$, where $d$ is the degree of $X$ with respect to $\mathcal{O}_{X}(1)$.

Proof. We shall prove our assertion by induction on $\operatorname{dim} X$. When $\operatorname{dim} X=2$, our assertion follows directly from Theorem 4.6, (1). Assume that $\operatorname{dim} X \geq 3$. Take the open set $U$ of $T_{0}$ and the filtration

$$
0=\tilde{E}_{0} \subseteq \tilde{E}_{1} \subseteq \cdots \sqsubseteq \tilde{E}_{\alpha}=\tilde{E}
$$

of Lemma 2.2. If $r\left(\tilde{E}_{i} / \tilde{E}_{i-1}\right)<\operatorname{dim} X-1$ for every $1 \leq i \leq \alpha$, then for general point $u$ of $U, 0=\left.\left.\left.\tilde{E}_{0}\right|_{\tilde{Y}_{u}} \subsetneq \tilde{E}_{1}\right|_{\tilde{Y}_{u}} \subsetneq \cdots \subseteq \tilde{E}_{\alpha}\right|_{\tilde{Y}_{u}}=\left.\tilde{E}\right|_{\tilde{Y}_{u}}$ is the HarderNarasimhan filtration of $\left.\tilde{E}\right|_{\tilde{Y}_{u}}=\left.E\right|_{\tilde{Y}_{u}}$ by virtue of Theorem 3.1. Thus, $E$ has a coherent subsheaf $E^{\prime}$ with $\mu\left(E^{\prime}\right)=\mu\left(\tilde{E}_{1} \otimes k(u)\right)>\mu(\tilde{E} \otimes k(u))=\mu(E)$ by virtue of Proposition 2.4. This can not happen because $E$ is $\mu$-semistable. Therefore, we see that $\alpha=2$ and $r\left(\tilde{E}_{1}\right)=1$ or $r(E)-1$. Assume that $r\left(\tilde{E}_{1}\right)=r(E)-1$. If for general $\tilde{Y}_{u},\left.\tilde{E}_{1}\right|_{\tilde{Y}_{u}}$ is $\mu$-semi-stable, then we see that $E$ is not $\mu$-semi-stable by the similar argument to the above. Fix a general member $Z$ of $L$ such that $\left.E\right|_{Z}$ has the Harder-Narasimhan filtration

$$
0=E_{0} \subsetneq E_{1} \subsetneq E_{2}=\left.E\right|_{Z}
$$

and for every general member $Y$ of $\operatorname{Tr}_{Z}(L),\left.E_{1}\right|_{Y}$ is not $\mu$-semi-stable. Applying the induction hypothesis to $\left(E_{1}, Z, L^{\prime}=\operatorname{Tr}_{Z}(L)\right),\left.E_{1}\right|_{Y}$ has the Harder-Narasimhan filtration for general $Y$ of $L^{\prime}$

$$
0=F_{0} \subsetneq F_{1} \subsetneq F_{2}=\left.E_{1}\right|_{Y}
$$

such that $r\left(F_{1}\right)=1$ or $r(E)-2$ and $-d \leq r\left(F_{1}\right) d\left(E_{1}, \mathcal{O}_{Z}(1)\right)-r\left(E_{1}\right) d\left(F_{1}, \mathcal{O}_{Y}(1)\right)$ $<0$. If $\mu\left(F_{2} / F_{1}\right)>\mu\left(E_{2} / E_{1}\right)$ for every general $Z$ and $Y$, the open set $W_{1}$ of Lemma 2.2 is not empty and hence, $E$ has a coherent subsheaf $E^{\prime}$ with $\mu\left(E^{\prime}\right)=\mu\left(E_{1}\right)>\mu\left(\left.E\right|_{z}\right)=\mu(E)$. This is not the case because $E$ is $\mu$ - 
semi-stable. Thus $\mu\left(F_{2} / F_{1}\right) \leq \mu\left(E_{2} / E_{1}\right)$. Setting $r=r(E)$, we have two cases:

Case I. $r\left(F_{1}\right)=r-2$; then we obtain

$$
(r-2) d\left(E_{1}\right)-(r-1) d\left(F_{1}\right) \leq-d \quad \text { and } \quad d\left(E_{1}\right)-d\left(F_{1}\right) \leq d(E)-d\left(E_{1}\right) .
$$

Thus $-d \leq(r-2) d\left(E_{1}\right)+(r-1) d(E)-2(r-1) d\left(E_{1}\right)=(r-1) d(E)-r d\left(E_{1}\right)$.

Case II. $r\left(F_{1}\right)=1$, then we get

$$
d\left(E_{1}\right)-(r-1) d\left(F_{1}\right) \leq-d \quad \text { and } \quad d\left(E_{1}\right)-d\left(F_{1}\right) \leq(r-2) d(E)-d\left(E_{1}\right) .
$$

Hence we have

$$
(r-1) d(E)-r d\left(E_{1}\right) \geq-d /(r-1) \geq-d
$$

The inequality $(r-1) d(E)-r d\left(E_{1}\right)<0$ follows from the fact that $E_{1}$ is a filter of the Harder-Narasimhan filtration of $\left.E\right|_{z}$.

In the case of $r\left(\tilde{E}_{1}\right)=1$, we can prove our assertion similarly to the above.

q.e.d.

In the case of $r(E)=\operatorname{dim} X+1$, a similar result is obtained.

LEMMA 5.3. Under the same situation as in Lemma 5.2, assume that $r(E)-1=\operatorname{dim} X \geq 2, E$ is $\mu$-semi-stable and that $\left.E\right|_{z}$ is not $\mu$-semi-stable with respect to $\mathcal{O}_{Z}(1)$ for every general $Z$ of $L$. Then, for general $Z$ of $L$, $\left.E\right|_{z}$ has the Harder-Narasimhan filtration

$$
0=E_{0} \subsetneq E_{1} \subsetneq \cdots \subsetneq E_{\alpha}=\left.E\right|_{Z}
$$

such that (1) $\mu(E)-d \leq \mu\left(E_{i} / E_{i-1}\right) \leq \mu(E)+d$ for $1 \leq i \leq \alpha, \quad d=$ $d\left(\mathcal{O}_{X}(1), \mathcal{O}_{X}(1)\right)$ and (2) one of the following properties (i) and (ii) is enjoyed;

(i) $\alpha=3$ and $\left(r\left(E_{1}\right), r\left(E_{2} / E_{1}\right), r\left(E / E_{2}\right)\right)$ is a permutation of $(1,1, r(E)-2)$,

(ii) $\alpha=2$ and $\left(r\left(E_{1}\right), r\left(E / E_{1}\right)\right)$ is a permutation of $(1, r(E)-1)$ or $(2, r(E)-2)$.

Proof. If we would give a complete proof, we should exhaust many cases. Thus we shall discuss only some typical cases. Our proof is by induction on $\operatorname{dim} X$. Assume that $\operatorname{dim} X=2$. Then $r(E)=3$. By Lemma 2.2, we have only to show the lemma for the generic member $Z$ of $L$. Let us consider the case where $\left.E\right|_{z}$ has the Harder-Narasimhan filtration

$$
\Phi: 0=E_{0} \subsetneq E_{1} \subsetneq E_{2} \subsetneq E_{3}=\left.E\right|_{z} \text {. }
$$

Set $\mu\left(E_{i} / E_{i-1}\right)=d\left(E_{i} / E_{i-1}\right)=m_{i}$. Applying Theorem 4.6 to $\bar{F}=E_{2}$, we see 
that $E / E_{2} \otimes E_{2}^{\vee}$ contains a subbundle $M$ with $d(M) \geq-d$. If $r(M)=2$, then $2 m_{3}-m_{1}-m_{2} \geq-d$ and if $r(M)=1$, then $m_{3}-m_{2} \geq-d$. Taking the dual filtration $\Phi^{\vee}$ of $\Phi$

$$
\Phi^{\vee}: 0 \sqsubseteq\left(E_{3} / E_{2}\right)^{\vee} \subsetneq\left(E_{3} / E_{1}\right)^{\vee} \sqsubseteq E_{3}^{\vee}=\left.E^{\vee}\right|_{z},
$$

we have that $m_{2}+m_{3}-2 m_{1} \geq-d$ or $m_{2}-m_{1} \geq-d$. Combining these with $m_{1}+m_{2}+m_{3}=3 \mu(E)$ and $m_{1}>m_{2}>m_{3}$, the desired inequalities $\mu(E)-d \leq \mu\left(E_{i} / E_{i-1}\right) \leq \mu(E)+d$ are obtained. The proof of the other cases is similar to the above and easier.

Assume that $\operatorname{dim} X>2$. By the same argument as in the proof of Lemma 5.2, for every general $Z$ in $L,\left.E\right|_{Z}$ has the Harder-Narasimhan filtration

$$
\Psi: 0=E_{0} \subsetneq E_{1} \subsetneq \cdots \sqsubseteq E_{\alpha}=\left.E\right|_{z}
$$

with either (i) or (ii). Let us consider the case where $\Psi$ has the property (i) and $r\left(E_{2} / E_{1}\right)=r(E)-2=\operatorname{dim} Z$. By virtue of Lemma 5.2, for every general $Y$ of $\operatorname{Tr}_{Z}(L), F=\left.\left(E_{2} / E_{1}\right)\right|_{Y}$ has the Harder-Narasimhan filtration

$$
0=F_{0} \subseteq F_{1} \subsetneq F_{2}=F
$$

with $\mu(F)<\mu\left(F_{1}\right) \leq \mu(F)+d$ and $\mu(F)-d \leq \mu\left(F_{1} / F_{2}\right)<\mu(F)$. Let

$$
0=G_{0} \sqsubseteq G_{1} \sqsubseteq \cdots \sqsubseteq G_{\beta}=\left.E_{2}\right|_{Y}
$$

be the Harder-Narasimhan filtration of $\left.E_{2}\right|_{Y}$.

Sublemma 5.4. $\mu\left(G_{\beta} / G_{\beta-1}\right) \geq \mu\left(F / F_{1}\right) \geq \mu(F)-d$.

Proof. Let $F_{1}^{\prime}$ be the inverse image of $F_{1}$ to $\left.E_{2}\right|_{Y}$. Then, the filtration $0=\left.\left.\left.E_{0}\right|_{Y} \subseteq E_{1}\right|_{Y} \subseteq F_{1}^{\prime} \subseteq E_{2}\right|_{Y}$ induces a filtration $0 \subseteq \bar{E}_{1} \subseteq \bar{F}_{1} \subseteq \bar{G}=G_{\beta} / G_{\beta-1}$. Since all the $\left.E_{1}\right|_{Y}, F_{1}^{\prime} /\left(\left.E_{1}\right|_{Y}\right) \cong F_{1}$ and $\left(\left.E_{2}\right|_{Y}\right) / F_{1}^{\prime} \cong F / F_{1}$ are $\mu$-semi-stable, we have that $d\left(\bar{E}_{1}\right) \geq r\left(\bar{E}_{1}\right) \mu\left(\left.E_{1}\right|_{Y}\right), \quad d\left(\bar{F}_{1} / \bar{E}_{1}\right) \geq r\left(\bar{F}_{1} / \bar{E}_{1}\right) \mu\left(F_{1}\right)$ and $d\left(\bar{G} / \bar{F}_{1}\right) \geq$ $r\left(\bar{G} / \bar{F}_{1}\right) \mu\left(F / F_{1}\right)$. On the other hand, $\mu\left(\left.E_{1}\right|_{Y}\right)>\mu(F)>\mu\left(F / F_{1}\right), \mu\left(F_{1}\right)>\mu(F)$ $>\mu\left(F / F_{1}\right)$ and $\mu\left(F / F_{1}\right) \geq \mu(F)-d$. Thus $d\left(G_{\beta} / G_{\beta-1}\right) \geq r\left(G_{\beta} / G_{\beta-1}\right) \mu\left(F / F_{1}\right) \geq$ $r\left(G_{\beta} / G_{\beta-1}\right)(\mu(F)-d)$.

q.e.d.

Now, if $\mu\left(G_{\beta} / G_{\beta-1}\right)>\mu\left(E_{3} / E_{2}\right)$, then $\left.E_{2}\right|_{Y}$ is a filter of the HarderNarasimhan filtration of $\left.E\right|_{Y}$, whence $W_{2}$ in Lemma 2.2 is not empty. Then, this contradicts the $\mu$-semi-stability of $E$ by Proposition 2.4. Therefore, $\mu(F)-d \leq \mu\left(G_{\beta} / G_{\beta-1}\right) \leq \mu\left(E_{3} / E_{2}\right)$. By a similar argument we see also that $\mu\left(E_{1}\right) \leq \mu(F)+d$. From these, we can easily deduce the 
required inequalities $\mu(E)-d \leq \mu\left(E_{i} / E_{i-1}\right) \leq \mu(E)+d$. The other cases of $\alpha=3$ can be proved by the same way. When $\Psi$ has the property (ii), a similar but easier argument is applicable by using Lemma 5.2 or our induction hypothesis.

q.e.d.

If $E$ is a torsion free coherent sheaf on a non-singular variety, then there is a torsion free coherent sheaf $E^{\prime}$ containing $E$ such that $E^{\prime}$ satisfies $\left(S_{2}\right)$ and $\operatorname{codim}\left(\operatorname{Supp}\left(E^{\prime} \mid E\right)\right) \geq 2$ (see the proof of Lemma 3.5). Such an $E^{\prime}$ is unique up to isomorphisms and, as a matter of fact, $E^{\prime}=$ $\left(E^{\vee}\right)^{\vee}$. $E^{\prime}$ is written $\varepsilon(E)$. For a family $\mathscr{F}$ of torsion free coherent sheaves on non-singular varieties, $\varepsilon \mathscr{F}$ denotes $\{\varepsilon(E) \mid E \in \mathscr{F}\}$.

LEMMA 5.4. Let $f: X \rightarrow S$ be a smooth, projective, geometrically integral morphism of noetherian schemes with $\operatorname{dim} X / S=n$ and let $\mathcal{O}_{X}(1)$ be an f-very ample invertible sheaf on $X$. Let $\mathscr{F}, \mathscr{F}_{1}$ and $\mathscr{F}_{2}$ be families of the classes of coherent sheaves on the fibres of $X$ over $S$. Assume the following;

(1) every member of $\mathscr{F}$ is an extension of a member of $\mathscr{F}_{2}$ by a member of $\mathscr{F}_{1}$,

(2) every member of $\mathscr{F}_{1}$ and $\mathscr{F}_{2}$ is torsion free,

(3) every member of $\mathscr{F}$ satisfies the condition $\left(S_{2}\right)$,

(4) both $\mathscr{F}_{1}$ and $\varepsilon \mathscr{F}_{2}$ are bounded,

(5) $\left\{a_{2}(E) \mid E \in \mathscr{F}_{2}\right\}$ is a bounded set.

Then, $\mathscr{F}$ is bounded.

Proof. Take a member $E$ of $\mathscr{F}$. Then we have an exact sequence

$$
\Phi: 0 \longrightarrow E_{1} \longrightarrow E \longrightarrow E_{2} \longrightarrow 0
$$

with $E_{1} \in \mathscr{F}_{1}$ and $E_{2} \in \mathscr{F}_{2}$. Assume that $E, E_{1}$ and $E_{2}$ are on a fibre $X_{s}$. There exists a sequence $Y_{1}, \cdots, Y_{n-2}$ of members of $\left|\mathcal{O}_{X_{s}}(1)\right|$ such that for every $0 \leq i \leq n-2, Z_{i}=Y_{1} \cdots Y_{i}\left(Z_{0}=X_{s}\right)$ has the following properties; (a) $Z_{i}$ is smooth and $\operatorname{dim} Z_{i}=n-i$, (b) $\left.E\right|_{Z_{i}}$ satisfies $\left(S_{2}\right)$, (c) $\left.E_{1}\right|_{Z_{i}},\left.E_{2}\right|_{Z_{i}}$ and $\left.\varepsilon\left(E_{2}\right)\right|_{z_{i}}$ are torsion free, (d) the restriction of the exact sequence $\Phi$ to $Z_{i}$ is also exact and (e) $\left.\varepsilon\left(E_{2}\right)\right|_{z_{i}}=\varepsilon\left(\left.E_{2}\right|_{Z_{i}}\right)$. Set $\mathscr{F}^{(i)}=\left\{\left.E\right|_{Z_{i}} \mid E \in \mathscr{F}\right\}, \mathscr{F}_{1}^{(i)}$ $=\left\{\left.E_{1}\right|_{Z_{i}} \mid E \in \mathscr{F}\right\}, \mathscr{F}_{2}^{(i)}=\left\{\left.E_{2}\right|_{Z_{i}} \mid E \in \mathscr{F}\right\}$ and $\varepsilon\left(\mathscr{F}_{2}\right)^{(i)}=\left\{\left.\varepsilon\left(E_{2}\right)\right|_{Z_{i}} \mid E \in \mathscr{F}\right\}=\varepsilon_{\mathscr{F}_{2}}^{(i)}$. We shall prove the boundedness of $\mathscr{F}^{(i)}$ by descending induction on $i$. Since $\mathscr{F}_{1}$ and $\varepsilon \mathscr{F}_{2}$ are bounded, so are $\mathscr{F}_{1}^{(i)}$ and $\varepsilon\left(\mathscr{F}_{2}\right)^{(i)}$. Thus $\sharp\{\chi(F(m)) \mid F$ $\left.\in \mathscr{F}_{1}^{(i)}\right\}<\infty$ and $\sharp\left\{\chi(G(m)) \mid G \in \varepsilon\left(\mathscr{F}_{2}\right)^{(i)}\right\}<\infty$. On the other hand, $a_{0}(G)$ $=a_{0}(\varepsilon(G))$ and $a_{1}(G)=a_{1}(\varepsilon(G))$. Moreover, $a_{2}\left(E_{2}\right)=a_{2}\left(\left.E_{2}\right|_{Z_{i}}\right)$. Thus, in 
particular, $\left\{\chi(G(m)) \mid G \in \mathscr{F}_{2}^{(n-2)}\right\}<\infty$ by the assumption (5). Since every member of $\mathscr{F}_{2}^{(n-2)}$ is a subsheaf of a member of the bounded family $\varepsilon\left(\mathscr{F}_{2}\right)^{(n-2)}$, the above result implies that $\mathscr{F}_{2}^{(n-2)}$ is bounded. Therefore, $\mathscr{F}^{(n-2)}$ is bounded. Thus our assertion is proved for $i=n-2$. Assume that $\mathscr{F}^{(i)}$ is bounded for an $i \leq n-2$. Replacing $\mathscr{F}$ by $\mathscr{F}(m)=\{E(m) \mid E$ $\in \mathscr{F}\}, m \gg 0$, we may assume that (i) $H^{j}\left(Z_{i},\left(\left.E\right|_{Z_{i}}\right)(\ell)\right)=0$ for all $j \geq 1$, $\ell \geq 0$ and $E \in \mathscr{F}$, (ii) $\operatorname{dim} H^{0}\left(Z_{i},\left(\left.E\right|_{Z_{i}}\right)(\ell)\right) \leq c_{\ell}$, $\operatorname{dim} H^{1}\left(Z_{i},\left(\left.E\right|_{Z_{i}}\right)(\ell)\right) \leq c_{\ell}^{\prime}$ for all $E \in \mathscr{F}$, (iii) $\sharp\left\{\chi\left(\left(\left.E\right|_{Z_{i}}\right)(m)\right) \mid E \in \mathscr{F}\right\}<\infty$, (iv) there is a sequence of integers $(b)=\left(b_{1}, \cdots, b_{n-i+1}\right)$ such that $\left.E\right|_{Z_{i}}$ is a $(b)$-sheaf for all $E \in \mathscr{F}$ and (v) there is an integer $\ell_{0}$ such that $H^{0}\left(Z_{i},\left(\left.E\right|_{Z_{i}}\right)(-\ell)\right)=H^{1}\left(Z_{i},\left(\left.E\right|_{Z_{i}}\right)(-\ell)\right)$ $=0$ for all $\ell \geq \ell_{0}$. Since $H^{0}\left(Z_{i-1},\left(\left.E_{2}\right|_{Z_{i-1}}\right)(\ell)\right) \subseteq H^{0}\left(Z_{i-1},\left(\left.\varepsilon\left(E_{2}\right)\right|_{Z_{i}{ }_{1}}(\ell)\right)\right.$ by (e), $\left.\varepsilon\left(E_{2}\right)\right|_{Z_{i-1}}$ is torsion free by (c) and (e) and since $\varepsilon\left(\mathscr{F}_{2}\right)^{(i-1)}$ is bounded, we see that there is an integer $\ell_{1}$ such that $H^{0}\left(Z_{i-1}, G(-\ell)\right)=0$ for all $\ell$ $\geq \ell_{1}$ and $G \in \mathscr{F}_{2}^{(i-1)}$. On the other hand, since $\mathscr{F}_{1}^{(i-1)}$ is bounded and every member of $\mathscr{F}_{1}^{(i-1)}$ is torsion free, we may assume that $H^{0}\left(Z_{i-1}, F(-\ell)\right)$ $=0$ for all $F \in \mathscr{F}_{1}^{(i-1)}$ and $\ell \geq \ell_{1}$. Therefore, $H^{0}\left(Z_{i-1},\left(\left.E\right|_{Z_{i-1}}\right)(-\ell)\right)=0$ for all $E \in \mathscr{F}$ and $\ell \geq \ell_{1}$. Thanks to this and (i)-(v) above, we can employ the same argument as in the proof of Proposition 3.6 and know that $\mathscr{F}^{(i-1)}$ is bounded.

q.e.d.

Now, we are ready to prove the following.

Proposition 5.6. (1) If $B_{n-1, r}^{\prime}(\Lambda)$ holds and if $r \leq n-1$, then $B_{n, r}^{\prime}(\Lambda)$ holds.

(2) Assume that $\Lambda$ is a field of characteristic zero. If $B_{n-1, n}^{\prime}(\Lambda)$ and $B_{n-1, n-1}^{\prime}(\Lambda)$ hold, then so does $B_{n, n}^{\prime}(\Lambda)$.

(3) Assume that $\Lambda$ is a field of characteristic zero. If $B_{n-1, n-1}^{\prime}(\Lambda)$, $B_{n-1, n}^{\prime}(\Lambda)$ and $B_{n-1, n+1}^{\prime}(\Lambda)$ hold, then so does $B_{n, n+1}^{\prime}(\Lambda)$.

Proof. (1) is an immediate consequence of Theorem 3.1 and Proposition 3.6. To prove (2), let $\mathscr{F}$ be the subfamily of $\Sigma_{X / S}^{\prime}\left(n, n, a_{1}, a_{2}\right)$ such that $E$ on a fibre $X_{s}$ is contained in $\mathscr{F}$ if and only if $E$ is a member of $\Sigma_{X / S}^{\prime}\left(n, n, a_{1}, a_{2}\right)$ and for general $Y$ in $\left|\mathcal{O}_{X_{s}}(1)\right|,\left.E\right|_{Y}$ is $\mu$-semi-stable. Since $B_{n-1, n}^{\prime}(\Lambda)$ holds, $\mathscr{F}$ is bounded by virtue of Proposition 3.6. If $E$ is contained in $\mathscr{F}^{\prime}=\Sigma_{X / S}^{\prime}\left(n, n, a_{1}, a_{2}\right)-\mathscr{F}$, then $\left.E\right|_{Z}$ is not $\mu$-semi-stable for every general member $Z$ in $\left|\mathcal{O}_{X_{s}}(1)\right|$. Lemma 5.2 provides us with the Harder-Narasimhan filtration

$$
0=\left.E_{0} \subsetneq E_{1} \subsetneq E\right|_{Z}
$$


for general $Z \in\left|\mathcal{O}_{X_{s}}(1)\right|$ such that $r\left(E_{1}\right)=1$ or $r(E)-1$ and $-d \leq$ $r\left(E_{1}\right) d\left(E, \mathcal{O}_{X_{s}}(1)\right)-r(E) d\left(E_{1}, \mathcal{O}_{Z}(1)\right)<0$, where $d$ is the maximum of the degrees of $X_{s}$ with respect to $\mathcal{O}_{X_{s}}(1)$. We may assume that $\left.E\right|_{Z}$ satisfies the condition $\left(S_{2}\right)$ (Lemma 3.7). Since $a_{1}(E)$ is fixed, $\left\{a_{1}\left(E_{1}\right) \mid E \in \mathscr{F}^{\prime}\right\}$ is bounded. Setting $E_{2}=\left(\left.E\right|_{z}\right) / E_{1}$, we have the exact sequence

$$
\left.0 \longrightarrow E_{1} \longrightarrow E\right|_{Z} \longrightarrow E_{2} \longrightarrow 0
$$

and $\left\{a_{1}\left(E_{2}\right) \mid E \in \mathscr{F}^{\prime}\right\}$ is bounded. Now, let us consider two families $\mathscr{G}_{1}=$ $\left\{E_{1} \mid E \in \mathscr{F}^{\prime}\right\}$ and $\mathscr{G}_{2}=\left\{E_{2} \mid E \in \mathscr{F}^{\prime}\right\}$. Since $\left\{a_{1}\left(E_{2}\right) \mid E_{2} \in \mathscr{G}_{2}\right\}$ is bounded and since $B_{n-1, n-1}^{\prime}(\Lambda)$ and $B_{n-1,1}^{\prime}(\Lambda)$ hold, $\left\{a_{2}\left(E_{2}\right) \mid E_{2} \in \mathscr{G}_{2}\right\}$ bounded from above by $b_{2}$ (Lemma 5.1). From this and the fact that $a_{2}\left(\left.E\right|_{z}\right)=a_{2}(E) \geq a_{2}$, we infer that $\left\{a_{2}\left(E_{1}\right) \mid E_{1} \in \mathscr{G}_{1}\right\}$ is bounded from below by $a_{2}-b_{2}$. Similarly, $\left\{a_{2}\left(E_{2}\right) \mid E_{2} \in \mathscr{G}_{2}\right\}$ is bounded from below by $a_{2}-b_{1}$. Therefore, we have

(5.6.1) $\left\{a_{1}\left(E_{1}\right) \mid E_{1} \in \mathscr{G}_{1}\right\},\left\{a_{2}\left(E_{1}\right) \mid E_{1} \in \mathscr{G}_{1}\right\},\left\{a_{1}\left(E_{2}\right) \mid E_{2} \in \mathscr{G}_{2}\right\}$

and $\left\{a_{2}\left(E_{2}\right) \mid E_{2} \in \mathscr{G}_{2}\right\}$ are bounded sets of integers.

Since $E_{2}$ is torsion free and $\left.E\right|_{Z}$ satisfies $\left(S_{2}\right), E_{1}$ does $\left(S_{2}\right)$. Thus we see that $\mathscr{G}_{1}$ is contained in a finite union of $\Sigma_{X^{\prime} / S^{\prime}}^{\prime}(n-1, n-1, a, b)$ 's and $\Sigma_{X^{\prime} / S^{\prime}}^{\prime}(n-1,1, a, b)^{\prime}$, where $X^{\prime} / S^{\prime}$ is the same as before Proposition 3.6. Therefore,

(5.6.2) $\mathscr{G}_{1}$ is bounded.

$\varepsilon\left(E_{2}\right)$ satisfies $\left(S_{2}\right), a_{1}\left(\varepsilon\left(E_{2}\right)\right)=a_{1}\left(E_{2}\right)$ and $a_{2}\left(\varepsilon\left(E_{2}\right)\right) \geq a_{2}\left(E_{2}\right)$ (see the proof of Lemma 3.5). Thus, for the same reason as $\mathscr{G}_{1}$, we have

(5.6.3) $\varepsilon\left(\mathscr{G}_{2}\right)$ is bounded.

By virtue of (5.6.1), (5.6.2) and (5.6.3), we can apply Lemma 5.5 to $\left\{\left.E\right|_{z} \mid E \in \mathscr{F}^{\prime}\right\}$ and we see that $\left\{\left.E\right|_{z} \mid E \in \mathscr{F}^{\prime}\right\}$ is bounded. Then the same argument as in the proof of Proposition 3.6 shows that $\mathscr{F}^{\prime}$ is bounded. We see therefore that $\Sigma_{X / S}^{\prime}\left(n, n, a_{1}, a_{2}\right)=\mathscr{F} \cup \mathscr{F}^{\prime}$ is bounded.

Next, let us prove (3). Let $\mathscr{F}$ and $\mathscr{F}^{\prime}$ be the subfamilies of $\Sigma_{X / S}^{\prime}\left(n, n+1, a_{1}, a_{2}\right)$ defined similarly to $\mathscr{F}$ and $\mathscr{F}^{\prime}$ in the proof of (2). For the same reason as (2), $\mathscr{F}$ is bounded. By virtue of Lemma 5.3, we have the Harder-Narasimhan filtration

$$
0=E_{0} \subsetneq E_{1} \subsetneq \cdots \sqsubseteq E_{\alpha}=\left.E\right|_{z}
$$

for $E \in \mathscr{F}^{\prime}$ and general $Z \in\left|\mathcal{O}_{X_{s}}(1)\right|$ with the properties (1) and (2). As 
before, we may assume that $\left.E\right|_{z}$ satisfies $\left(S_{2}\right)$. By the property (1), $\left\{a_{1}\left(E_{i}\right) \mid E \in \mathscr{F}^{\prime}\right\}$ is bounded. Since $B_{n-1,1}^{\prime}(\Lambda), B_{n-1,2}^{\prime}(\Lambda), B_{n-1, n-1}^{\prime}(\Lambda)$ and $B_{n-1, n}^{\prime}(\Lambda)$ hold, we see that $\left\{a_{2}\left(E_{\alpha} / E_{\alpha-1}\right) \mid E \in \mathscr{F}^{\prime}\right\}$ is bounded from above, whence $\left\{a_{2}\left(E_{\alpha-1}\right) \mid E \in \mathscr{F}^{\prime}\right\}$ is bounded from below. Repeating this procedure, it is seen that $\left\{a_{2}\left(E_{1}\right) \mid E \in \mathscr{F}^{\prime}\right\}$ is a bounded set. Hence, $\left\{a_{2}\left(E_{2} / E_{1}\right) \mid E \in \mathscr{F}^{\prime}\right\}$ is bounded and so on. Thus we have

(5.6.4) $\left\{a_{1}\left(E_{i} / E_{i-1}\right) \mid E \in \mathscr{F}^{\prime}\right\}$ and $\left\{a_{2}\left(E_{i} / E_{i-1}\right) \mid E \in \mathscr{F}^{\prime}\right\}$ are bounded.

Set $\mathscr{H}_{1}=\left\{E_{\alpha-1} \mid E \in \mathscr{F}^{\prime}\right\}, \mathscr{H}_{2}=\left\{E_{\alpha}\left|E_{\alpha-1}\right| E \in \mathscr{F}^{\prime}\right\}, \mathscr{H}_{1}^{\prime}=\left\{E_{1} \mid E \in \mathscr{F}^{\prime}\right\}$ and $\mathscr{H}_{1}^{\prime \prime}=\left\{E_{\alpha-1} / E_{1} \mid E \in \mathscr{F}^{\prime}\right\}$. Since every member of $\mathscr{H}_{2}$ is torsion free and $\left.E\right|_{z}$ satisfies $\left(S_{2}\right)$, every member of $\mathscr{H}_{1}$ satisfies $\left(S_{2}\right)$ and hence, so does every member of $\mathscr{H}_{1}^{\prime}$. Since $B_{n-1,1}^{\prime}(\Lambda), B_{n-1,2}^{\prime}(\Lambda), B_{n-1, n-1}^{\prime}(\Lambda)$ and $B_{n-1, n}^{\prime}(\Lambda)$ hold, we see that $\mathscr{H}_{1}^{\prime}$ and $\varepsilon\left(\mathscr{H}_{1}^{\prime \prime}\right)$ is bounded by (5.6.4). Since each member of $\mathscr{H}_{1}$ is an extension of a member of $\mathscr{H}_{1}^{\prime \prime}$ by a member of $\mathscr{H}_{1}^{\prime}, \mathscr{H}_{1}$ is bounded by virtue of (5.6.4) and Lemma 5.5. Then, applying Lemma 5.5 to $\mathscr{F}_{1}=$ $\mathscr{H}_{1}, \quad \mathscr{F}=\left\{\left.E\right|_{z} \mid E \in \mathscr{F}^{\prime}\right\}$ and $\mathscr{F}_{2}=\mathscr{H}_{2}$, we know that $\left\{\left.E\right|_{z} \mid E \in \mathscr{F}^{\prime}\right\}$ is bounded. Therefore, $\mathscr{F}^{\prime}$ is bounded similarly to (2). Thus, $\Sigma_{X / S}^{\prime}\left(n, n+1, a_{1}, a_{2}\right)$ $=\mathscr{F} \cup \mathscr{F}^{\prime}$ is bounded.

q.e.d.

As a direct corollary to Proposition 5.6, we have

THEOREM 5.7. If $\Lambda$ is a field of characteristic zero, then $B_{n, 3}^{\prime}(\Lambda)$ and $B_{n, 4}^{\prime}(\Lambda)$ hold for all $n$ and hence, so do $B_{n, 3}^{\prime \prime}(\Lambda)$ and $B_{n, 4}^{\prime \prime}(\Lambda)$ (for the notation, see (3.4.2) and (3.4.3)).

Proof. $\quad B_{1,3}^{\prime}(\Lambda)$ and $B_{1,4}^{\prime}(\Lambda)$ are well-known and $B_{2,3}^{\prime}(\Lambda)$ and $B_{2,4}^{\prime}(\Lambda)$ are special cases of Theorem 3.10. Assume that $n \geq 3$ and $B_{n-1,3}^{\prime}(\Lambda)$ and $B_{n-1,4}^{\prime}(\Lambda)$ hold. Since $B_{n-1,2}^{\prime}(\Lambda)$ holds by Theorem 3.11, Proposition 5.6 implies that $B_{n, 3}^{\prime}(\Lambda)$ and $B_{n, 4}^{\prime}(\Lambda)$ hold. Therefore, by induction on $n$, we see that $B_{n, 3}^{\prime}(\Lambda)$ and $B_{n, 4}^{\prime}(\Lambda)$ hold for all $n$.

q.e.d.

Remark 5.8. (1) To remove the restriction on $\Lambda$ from Theorem 5.7, we should replace Lemma 5.2 and Lemma 5.3 by suitable lemmas. If the characteristic is positive, Lemma 5.2 and Lemma 5.3 are false as was shown in Remark 4.7. The author has a result which can play the same role in the proof of Proposition 5.6 as Lemma 5.2. Thus Proposition 5.6, (1), (2) and hence $B_{n, 3}^{\prime}(\Lambda)$ hold for all $\Lambda$ and $n$. But almost all the parts of its proof consist of complicated computations. Hence, I will write it elsewhere. 
(2) The proof of Proposition 5.6 shows that $B_{n, r}^{\prime}(\Lambda)$ holds for all $n$ and $r$ if and only if the following statement $M_{n, r}(\Lambda)$ holds for all $n$ and $r$.

$M_{n, r}(\Lambda)$ : Let $E$ on a fibre $X_{s}$ be a member of $\Sigma_{X / S}^{\prime}\left(n, r, a_{1}, a_{2}\right)$. If $\left.E\right|_{Z}$ is not $\mu$-semi-stable for almost all $Z$ in $\left|\mathcal{O}_{X_{s}}(1)\right|$, then for sufficiently general members $Z$ of $\left|\mathcal{O}_{X_{s}}(1)\right|,\left.E\right|_{Z}$ has the Harder-Narasimhan filtration

$$
0=E_{0} \subsetneq E_{1} \subsetneq \cdots \subsetneq E_{\alpha}=\left.E\right|_{Z}
$$

such that $\mu(E)-\nu \leq \mu\left(E_{i} / E_{i-1}\right) \leq \mu(E)+\nu$ with $\nu$ a constant depending only on the family $\Sigma_{X / S}^{\prime}\left(n, r, a_{1}, a_{2}\right)$.

Theorem 3.1, Lemma 5.2 and Lemma 5.3 show that $M_{n, r}(\Lambda)$ holds with $\nu=d$ if $r \leq n+1$ and $A$ is a field of characteristic zero.

Let $\bar{M}_{X / S}(H)$ be the moduli scheme of semi-stable sheaves obtained in Theorem 4.11 of [10]. Corollary 5.9.1 of [10], Theorem 3.10, Theorem 3.11 and Theorem 5.7 provide us with the following

Theorem 5.9. (1) If $\operatorname{dim} X / S \leq 2$, then $\bar{M}_{X / S}(H)$ is a projective scheme over $S$.

(2) If the rank is two, then $\bar{M}_{X / S}(H)$ is a projective scheme over $S$.

(3) If $S$ is a scheme over a field of characteristic zero and if the rank is three or four, then $\bar{M}_{X / S}(H)$ is a projective scheme over $S$.

\section{REFERENCES}

[1] M. F. Atiyah, Vector bundles over an elliptic curve, Proc. London Math. Soc. (3) 7, 1957.

[2] W. Barth, Some properties of stable rank-2 vector bundles on $\boldsymbol{P}_{n}$, Math. Ann., 226, 1977.

[ 3 ] D. Gieseker, On the moduli of vector bundles on an algebraic surface, Ann. of Math., 106, 1977.

[4] A. Grothendieck, Technique de construction et théorème d'existence en géométrie algébrique, IV: Les schémas de Hilbert, Sem. Bourbaki, t. 13, 1960/61, nº 221.

[EGA] A. Grotendieck and J. Dieudonne, Éléments de Géométrie Algébrique, Chap. IV, Publ. Math. I. H. E. S., Nos. 20, 24, 28 and 32.

[5] G. Harder and M. S. Narasimhan, On the cohomology groups of moduli spaces of vector bundles on curves, Math. Ann., 212, 1975.

[6] S. Kleiman, Les théorèmes de finitude pour le foncteur de Picard, Sem. de Géométrie Algébrique de Bois Marie, 1966/67, Expose XIII, Lect. Notes in Math., 225, Springer-Verlag, 1971.

[ 7 ] M. Maruyama, Stable vector bundles on an algebraic surface, Nagoya Math. J., $58,1975$.

[ 8 ] - Openness of a family of torsion free sheaves, J. Math. Kyoto Univ., 16, 1976. 
[ 9 ] —-, Moduli of stable sheaves, I, J. Math. Kyoto Univ., 17, 1977.

[10] - Moduli of stable sheaves, II, J. Math. Kyoto Univ., 18, 1978.

[11] J.-P. Serre, Faisceaux algébriques cohérents, Ann. of Math., 61, 1955.

[12] S. Shatz, The decomposition and specialization of algebraic families of vector bundles, Compositio Math., 35, 1977.

[13] F. Takemoto, Stable vector bundles on algebraic surfaces, Nagoya Math. J., 47, 1972.

Department of Mathematics

Kyoto University 